\title{
Super-resolved imaging of a single cold atom on a nanosecond timescale
}

\author{
Zhong-Hua Qian, ${ }^{1,2}$ Jin-Ming Cui, ${ }^{1,2, *}$ Xi-Wang Luo, ${ }^{3}$ Yong-Xiang Zheng, ${ }^{1,2}$ Yun-Feng \\ Huang, ${ }^{1,2, \dagger}$ Ming-Zhong Ai, ${ }^{1,2}$ Ran He, ${ }^{1,2}$ Chuan-Feng Li, ${ }^{1,2,}{ }^{\ddagger}$ and Guang-Can Guo ${ }^{1,2}$ \\ ${ }^{1}$ CAS Key Laboratory of Quantum Information, University of Science and Technology of China, Hefei 230026, China \\ ${ }^{2}$ CAS Center For Excellence in Quantum Information and Quantum Physics, \\ University of Science and Technology of China, Hefei 230026, China \\ ${ }^{3}$ Department of Physics, The University of Texas at Dallas, Richardson, Texas 75080-3021, USA
}

(Dated: November 23, 2021)

\begin{abstract}
In cold atomic systems, fast and high-resolution microscopy of individual atoms is crucial, since it can provide direct information on the dynamics and correlations of the system. Here, we demonstrate nanosecond-scale two-dimensional stroboscopic pictures of a single trapped ion beyond the optical diffraction limit, by combining the main idea of ground-state depletion microscopy with quantum state transition control in cold atoms. We achieve a spatial resolution up to $175 \mathrm{~nm}$ using an NA = 0.1 objective in the experiment, which represents a more than tenfold improvement compared with direct fluorescence imaging. To show the potential of this method, we apply it to observe the secular motion of the trapped ion, we demonstrate a temporal resolution up to $50 \mathrm{~ns}$ with a displacement detection sensitivity of $10 \mathrm{~nm}$. Our method provides a powerful tool for probing particle positions, momenta, and correlations, as well as their dynamics in cold atomic systems.
\end{abstract}

Introduction. - Cold atomic systems, including cold quantum gases in optical lattices, neutral atoms in optical tweezers, and trapped ions, are promising platforms to study quantum simulation, computation, and information processing. High-resolution optical detection and imaging of individual particles in these systems are essential procedures, since they can provide direct information on quantum phenomena (e.g., transport, correlations, and phase transitions). In recent decades, many microscope techniques have been developed for cold quantum gases $[1,2]$ and individual atoms in optical tweezers $[3,4]$ or ion traps [5-7]. However, the resolution of these methods is fundamentally restricted to the wavelength scale set by the optical diffraction limit, making them unsuitable for probing quantum phenomena related to the details of the wave function in a variety of many-body systems.

Meanwhile, optical super-resolved microscopy has developed to maturity as a powerful tool in chemistry and biology [8]. It allows chemical reactions and biological processes to be viewed on a nanometer scale $[9,10]$. In recent years, this method has also been applied to quantum systems, such as to imaging of solid-spin systems [11-14] and to tracking of single-ion positions $[6,15,16]$, and it has been developed further in combination with quantum techniques for specific systems [17-21]. Realization of detection and manipulation below the subwavelength scale in cold atomic systems would allow the study of quantum transport, correlation, and dynamical phenomena in unprecedented ways, and therefore much research effort has been focused on this area, with substantial progress being made in recent years. Examples include the generation of nanoscale optical potentials [22-24], stroboscopic painting of optical potentials for atoms with subwavelength resolution [25, 26], and observation of the wave function of ensembles of atoms in one-dimensional optical lattices based on nonlinear atomic responses $[27,28]$ and pulsed ion microscopy [29]. However, two-dimensional (2D) super-resolved detection of a single cold atom below the optical diffraction limit has yet to be demonstrated.

Here we present a demonstration of ground-state depletion (GSD) microscopy on a trapped ion system to achieve 2D imaging beyond the optical diffraction limit. By combining the control sequence of the GSD microscopy with the qubit states polarization and detection methods for the ion, a 2D image of a trapped ion with a resolution of $175 \mathrm{~nm}$ is experimentally realized using an NA $=0.1$ imaging objective, which is a 13-fold improvement compared with direct fluorescence imaging. Our microscopy method not only enables single-particle imaging of cold atom systems to an unprecedented resolution, but, more importantly, it also has the advantage of high temporal resolution. The depletion process can be very short (around $50 \mathrm{~ns}$ ) under strong laser power, which enables us to take images in nanosecond intervals and thus allows the measurement of atomic wave-function dynamics. To show the capability of our method, we apply it to detect the secular motion of the trapped ion. One cycle of the motion within $735 \mathrm{~ns}$ is observed, with a displacement detection sensitivity of $10 \mathrm{~nm}$. Finally, we should point out that our method is quite general and can be applied to both ions and neutral atoms, and it can also be generalized to probe the many-body position correlations and interacting dynamics of atomic arrays by generating many independent depletion laser spots.

Experiment and results. - The imaging process is based on the transitions between ground states ${ }^{2} \mathrm{~S}_{1 / 2}$ and first excitation states ${ }^{2} \mathrm{P}_{1 / 2}$ of a ${ }^{171} \mathrm{Yb}^{+}$ion in a Paul trap (the transition wavelength is $369.5 \mathrm{~nm}$ ), which are sequentially controlled by different near-resonant lasers. The energy level diagram of ${ }^{171} \mathrm{Yb}^{+}$is presented in Fig. 1(a). Three different lasers are primarily used for the 
(a)

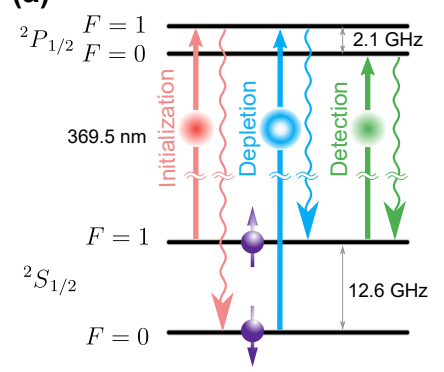

(c)

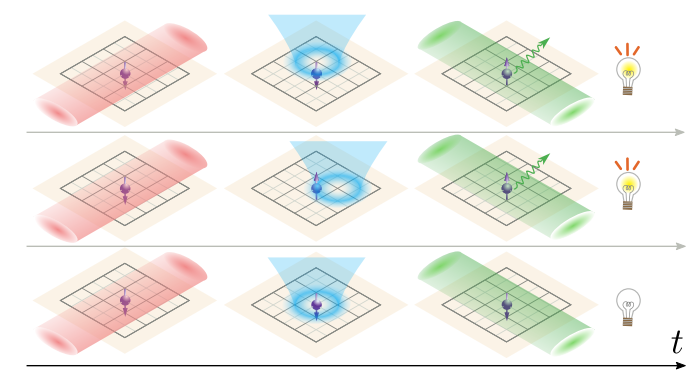

FIG. 1. Experimental scheme and setup. (a) Experimental scheme of energy levels of ${ }^{171} \mathrm{Yb}^{+}$. The ion is optically pumped by a Gaussian beam (red line) and initialized to ${ }^{2} \mathrm{~S}_{1 / 2}|\mathrm{~F}=0\rangle$ (dark state). Depletion light (blue line) with a donut shape pumps the spin into ${ }^{2} \mathrm{~S}_{1 / 2}|\mathrm{~F}=1\rangle$ (bright state). The detection laser (blue line) is used to read out the ion's state with state-dependent fluorescence. (b) Experimental setup: a Paul trap in a vacuum chamber is used to trap the ion, the initialization beam (red cylinder) and detection beam (green cylinder) are directed from the side windows of the vacuum chamber, and the collimated donut beam is focused by the imaging objective $(\mathrm{NA}=0.1)$. (c) Schematic of the super-resolved imaging process, in which three steps in (a) are performed to obtain a pixel of the image. A super-resolved image is obtained by scanning the donut spot, and only when the center of the donut spot is aligned with the ion does the ion avoid being polarized to the bright state and remains in the dark state.

imaging process: the initialization laser resonates with ${ }^{2} \mathrm{~S}_{1 / 2}|\mathrm{~F}=1\rangle \leftrightarrow{ }^{2} \mathrm{P}_{1 / 2}|\mathrm{~F}=1\rangle$, which is used to polarize the nuclear spin states of the ion to ${ }^{2} \mathrm{~S}_{1 / 2}|\mathrm{~F}=0\rangle$ (dark state); the depletion laser nearly resonates with ${ }^{2} \mathrm{~S}_{1 / 2}|\mathrm{~F}=0\rangle \leftrightarrow{ }^{2} \mathrm{P}_{1 / 2}|\mathrm{~F}=1\rangle$, which is used to depopulate the dark state to ${ }^{2} \mathrm{~S}_{1 / 2}|\mathrm{~F}=1\rangle$ (bright state); the detection laser, with ${ }^{2} \mathrm{~S}_{1 / 2}|\mathrm{~F}=1\rangle \leftrightarrow{ }^{2} \mathrm{P}_{1 / 2}|\mathrm{~F}=0\rangle$, is used to detect nuclear spin in a dark or bright state. As the transition between ${ }^{2} \mathrm{~S}_{1 / 2}|\mathrm{~F}=0\rangle$ and ${ }^{2} \mathrm{P}_{1 / 2}|\mathrm{~F}=0\rangle$ is forbidden, the state $\mathrm{S}_{1 / 2}|\mathrm{~F}=1\rangle$ can continually scatter fluorescence photons under the detection laser, which acts as a bright state; by contrast, the state $\mathrm{S}_{1 / 2}|\mathrm{~F}=0\rangle$ will not scatter detection photons, since it is not resonant with the detection laser, and acts as a dark state [30]. In the depletion process, a donut-shaped beam is focused on the ion to polarize its spin state through spontaneous emission. The ion's nuclear spin state is selectively polarized by scanning the donut spot position, allowing the position information to be encoded into nuclear spin states.
Figure 1(b) shows the configuration of the three lasers in the experimental setup. The ${ }^{171} \mathrm{Yb}^{+}$ion is trapped in a Paul trap installed in a vacuum chamber [31], and a magnetic field of $9.7 \mathrm{G}$ is applied along the $z$ axis. The initialization laser and detection laser are directed to the trap from the side windows of the chamber. These are Gaussian beams perpendicular to the magnetic field with waists of $30 \mu \mathrm{m}$. The depletion light beam is in a donut form and, along with the magnetic field, is focused to a donut spot through an objective lens with $\mathrm{NA}=0.1$, and then shone on the trapped ion across the top window of the vacuum chamber.

Figure 1(c) illustrates the main control sequence for the imaging process. The image is obtained pixel by pixel by scanning the depletion spot position on a $2 \mathrm{D}$ plane and sequentially switching the three lasers on and off. To illustrate the basic principle of obtaining an image of the ion, here we take just three pixels as an example. Only when the center of the donut spot is aligned with the ion does the ion avoid being polarized to the bright state in the depletion process and remains in the dark state. To keep the ion at a low temperature during the imaging process, we apply a $10 \mathrm{MHz}$ red detuned laser containing frequencies of ${ }^{2} \mathrm{~S}_{1 / 2}|\mathrm{~F}=1\rangle \leftrightarrow{ }^{2} \mathrm{P}_{1 / 2}|\mathrm{~F}=0\rangle$ and ${ }^{2} \mathrm{~S}_{1 / 2}|\mathrm{~F}=0\rangle \leftrightarrow{ }^{2} \mathrm{P}_{1 / 2}|\mathrm{~F}=1\rangle$ for Doppler cooling [32], and switch it on for $1 \mathrm{~ms}$ cooling before each pixel imaging sequence [33].

To realize high-spatial-resolution imaging in GSD, a highly focused donut spot with high mode purity is crucial, requiring the dark center's residual intensity to be as low as possible. To generate a donut spot with high purity, we employ a holographic beam reshaping method with a digital micromirror device (DMD) [34]. Compared with other methods [35-43], we can measure the optical system aberration in situ and then compensate for the aberration by this method. Our study first uses the trapped ion as a probe to detect the interference light intensity and measure the aberration phase map of the optical system in combination with the DMD [44]. Subsequently, computer-generated holograph patterns with aberration compensation are calculated and then programmed on the DMD to generate the desired beam profiles. To characterize the quality of focused spots after aberration compensation, we investigate the profiles of reshaped Gaussian and donut spots [see Figs. 2(a) and 2(b), respectively] by scanning the spots around the ion and detecting the ion's fluorescence with a cooling laser. The Gaussian spot is focused to a full width at half maximum (FWHM) of $2.34 \mu \mathrm{m}$, which is at its diffraction limit. For the donut beam, the residual light intensity in the dark center is $3.8 \%$ of the maximum intensity.

After preparation of the dark-center donut spot, the imaging sequences in Fig. 1(c) were applied with a linearly polarized donut beam. A super-resolved image of a single trapped ion was obtained, as shown in Fig. 2(c). The FWHM in the $x$ axis is $175 \mathrm{~nm}$ at $65 \mathrm{nW}$ deple- 

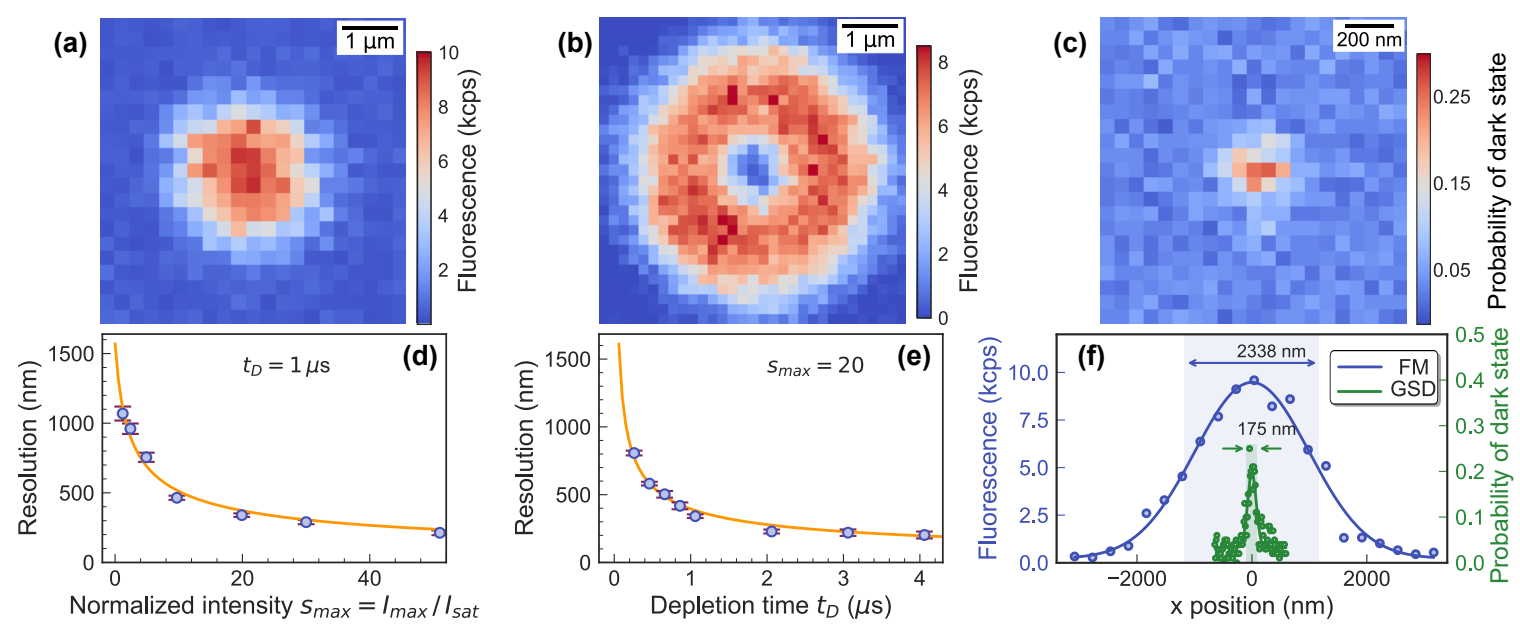

FIG. 2. Demonstration of super-resolved imaging of a trapped ion. (a) and (b) Use of the trapped ion as a probe to characterize the profile of Gaussian and donut spots, respectively, after aberration compensation using DMD with the holographic beam reshaping method [34]. The intensity is represented by the number of scattered fluorescence photons measured per second (kilocounts per second, kcps). The minimum intensity of the donut spot in the center is $3.8 \%$ of the maximum intensity. (c) Super-resolved image of a single ion. The depletion laser is linearly polarized, and the probability of the dark state is obtained from the statistics of 100 measurements. (d) Spatial resolution (FWHM) as a function of normalized intensity $s_{\max }=I_{\text {max }} / I_{\text {sat }}$ of donut light for $1 \mu$ s depletion time. (e) Spatial resolution as a function of depletion time with $s_{\max }$ fixed at 20 . (f) Contrast of resolutions between fluorescence microscope and GSD microscope with depletion time $t_{D}=7 \mu s$ and depletion power $P=65 \mathrm{nW}$ $\left(s_{\max }=14\right)$. The solid lines are fits with Gaussian functions.

tion laser power and $7 \mu$ s depletion time. A comparison of the measured point spread functions (PSFs), shown in Fig. 2(f), reveals that this technique gives a 13 -fold improvement in spatial resolution over fluorescence microscopy. We also analyzed the resolution as a function of the intensity and pulse duration of the depletion light, and the results are shown in Figs. 2(d) and 2(e). Here, for generality, the intensity is characterized by the normalized intensity $s_{\max }=I_{\max } / I_{\mathrm{sat}}$, where $I_{\max }$ denotes the maximum intensity of the donut spot profile and $I_{\text {sat }}=510 \mathrm{~W} / \mathrm{m}^{2}$ is the saturation intensity of the $\mathrm{Yb}^{+}$ ion. The results are consistent with the spatial resolution $\Delta r$ of the simplified model [45]:

$$
\Delta r=\frac{\mathrm{FWHM}_{01}}{\sqrt{1+k s_{\max } t_{D}}}=\frac{\mathrm{FWHM}_{00}}{1.67 \sqrt{1+k s_{\max } t_{D}}},
$$

where the coefficient $k=\beta \sigma I_{\text {sat }} / \hbar \omega_{0}, k s_{\max }$ represents the depletion rate, $\mathrm{FWHM}_{01}=1.41 \mu \mathrm{m}$ is the FWHM of the central hollow area of the donut spot $\left(\mathrm{LG}_{0}^{1}\right.$ mode), $\mathrm{FWHM}_{00}=2.34 \mu \mathrm{m}$ is the FWHM of the Gaussian spot ( $\mathrm{LG}_{0}^{0}$ mode), $\beta$ is the branching ratio for the decay into the bright state, $\sigma$ is the cross-section [46], and $t_{D}$ is the depletion pulse duration. This model indicates that the resolution is determined by the product of $s_{\max }$ and $t_{D}$. Theoretically, the resolution can be extended to the subnanometer scale as long as we increase $s_{\max } t_{D}$. However, further improvement in resolution is limited by actual experiment conditions. First, the imperfection of the donut spot results in residual light in the center. As the donut power increases, the residual light in the center $s_{0}$ will be large enough to excite the spin and make the position information indistinguishable from the probability noise. The resolution limit [47] $\Delta r_{\text {limit }} \propto \lambda /(\mathrm{NA} \sqrt{\mathrm{ER}})$, where $\mathrm{ER}=I_{\max } / I_{0}$ is defined as the extinction ratio of the doughnut spot. Second, the spatial resolution is limited by the ion's wave-packet size after cooling, which is about $20 \mathrm{~nm}$, as given by the Doppler cooling limit in the present study.

Next, we will present a method to detect the dynamics of a single cold atom in a 2D plane with high precision. Because the ion is trapped in a harmonic trap, it undergoes secular oscillations in three axes [48]. Here, we select the oscillation along two RF needles (the $y$ axis) for the study, as shown in Fig. 3(a). To demonstrate this application, we first drive the ion resonantly with an electrical pulse to excite the oscillation along the $y$ axis after Doppler cooling. The electrical pulse provides a driving force in the form $F_{d} \sin \left(\omega_{d} t\right)$, which drives at a frequency $\omega_{d}$ and lasts for a time $t_{d}$. During the driving process, the cooling lasers are turned off to avoid motion dissipation. When the driving pulse ends, the ion is drived into a steady-state oscillation, and the amplitude of the oscillation is given by

$$
A=\frac{2 F_{d}}{m\left(\omega_{y}^{2}-\omega_{d}^{2}\right)} \sin \left[\frac{\left(\omega_{y}-\omega_{d}\right) t_{d}}{2}\right],
$$

where $\omega_{y}=2 \pi \times 1.36 \mathrm{MHz}$ is the oscillation frequency of the ion, $t_{d}=100 T=100 \cdot 2 \pi / \omega_{y}$, and $m$ is the ion mass. Near resonance $\left(\omega_{d} \approx \omega_{y}\right)$, we find that $A=F_{d} t_{d} / 2 m \omega_{y}$, which indicates that the amplitude of motion is propor- 
(a)

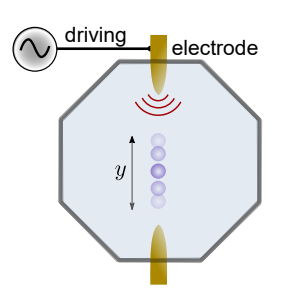

(b)

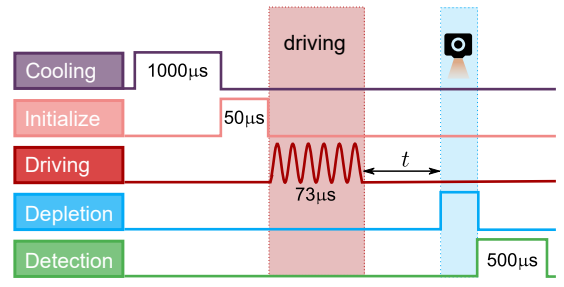

(d)

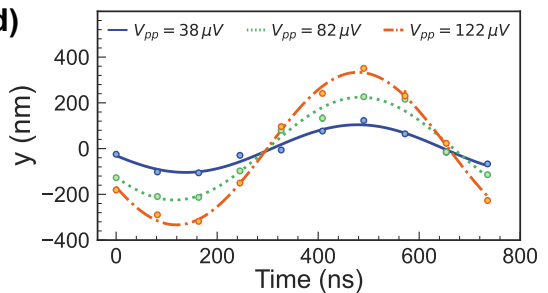

(c)
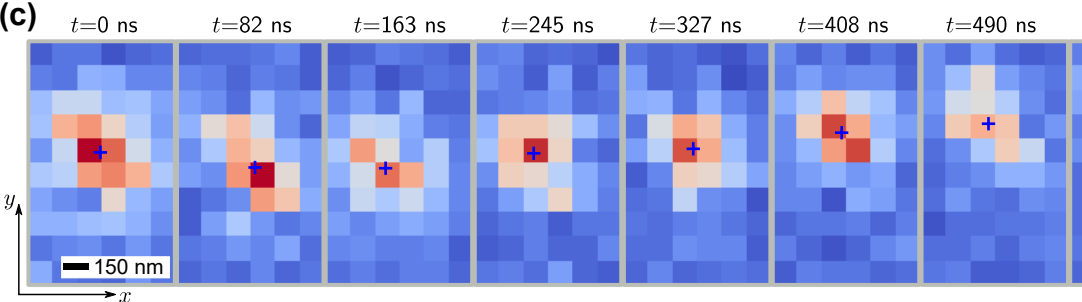

$t=572 \mathrm{~ns}$ $t=653 \mathrm{~ns}$ $t=735$ ns

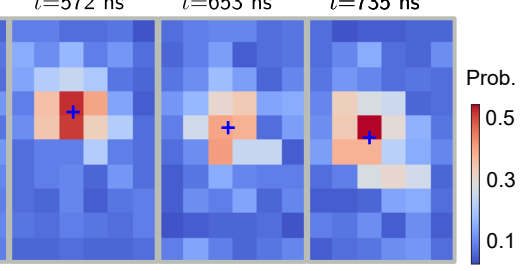

FIG. 3. Nanosecond photography to detect the dynamics of the trapped ion. (a) To excite the motion mode along the $y$ axis, a resonant electrical signal is applied to one electrode of the Paul trap, providing a sinusoidal driving force. (b) Sequence diagram of the imaging process. The ion is driven to steady oscillation by a resonant pulse, with a driving voltage $V_{\mathrm{pp}}$ and a driving duration of $73.5 \mu \mathrm{s}$. The ion is then imaged at $t$ after the driving has been stopped, with a 50 ns depletion duration. $s_{\text {max }}$ is fixed at 450. (c) Photographs of the moving ion driven by $V_{\mathrm{pp}}=38 \mu \mathrm{V}$ at different $t$. The blue crosses mark the fitted centers. (d) The ion's motional trajectories along the $y$ axis under different driving voltages, which are constructed by the fitted centers of super-resolved images.

tional to the driving force $F_{d}$ and driving time $t_{d}$.

To acquire the instantaneous position of the ion at time $t$ after the driving pulse, we implement the super-resolved microscopy with a delay time $t$ before the depletion process. To get a pixel in an image, a control sequence in Fig. 3(b) is repeated 100 times to get the dark state probability, which requires $0.16 \mathrm{~s}$. As the excited oscillation is synchronised to the driving wave phase, we fix the driving wave phase to make sure the current position of the ion is constant between different shots. To obtain one image, the donut beam is scanned to $10 \times 10$ pixels, while the depletion pulse duration is kept at $50 \mathrm{~ns}$, which is much shorter than the period of motion (735 ns). The normalized intensity of the doughnut spot $s_{\max }$ is fixed at 450 . The total time to get an image is $16 \mathrm{~s}$. By imaging the ion at different $t$, we can see its dynamics in the trap. Figure 3(c) shows a typical photographic recording of the oscillating ion in the central $6 \times 10$ pixels. By fitting each image to obtain the displacements of the ion at different $t$, the ion trajectory can be reconstructed with a precision of $10 \mathrm{~nm}$. The fitted spatial resolution is averaged as $373 \mathrm{~nm}$. The ion's oscillating trajectories under different driving forces are also investigated in this study, as shown in Fig. 3(d). The result shows that the amplitude of motion depends linearly on the driving force, as predicted by Eq. (2). We observed an amplitude of motion of $104.2 \pm 8.9 \mathrm{~nm}$ under a $38 \mu \mathrm{V}$ peak-to-peak driving voltage $\left(V_{\mathrm{pp}}=38 \mu \mathrm{V}\right)$. The corresponding driving force $F_{d}=6.9 \pm 0.7 \mathrm{zN}$. The period of motion is fitted as $681.1 \pm 23.9 \mathrm{~ns}$, which has a $7 \%$ error relative to the calculated period.

Outlook and conclusions. - In this work, we have demonstrated a nanosecond-scale super-resolved 2D imaging method for a single trapped ion. Our approach is quite general and can be applied to both charged atoms (e.g., the trapped ion studied here) and neutral ultracold atoms (e.g., a single atom trapped in an optical tweezer), for which the holographic beam reshaping method with DMD [34] as well as (nuclear-) spin polarization and detection have already been widely used. Moreover, an arbitrary array of spots with various shapes can be easily generated at the same time, using the holographic beam reshaping method [34], and each of them can be controlled independently. Therefore, by applying these depletion spots in parallel on site-resolved cold atoms (e.g., tweezer arrays), we can develop this imaging method to probe two- or multisite correlations and their interacting many-body dynamics.

It is worth noting that the resolution of the microscopy can be improved by using higher-NA objectives under the same depletion parameters (e.g., the NAs of objectives have reached 0.6 for trapped ion systems [6] and 0.7 for cold neutral atoms in optical tweezers [3]), and the GSD schemes will support an imaging resolution below $30 \mathrm{~nm}$ and a displacement measurement accuracy below $2 \mathrm{~nm}$ in principle, which is below the level of the wave-packet size of the motional ground state. Furthermore, the limit of resolution is approximately $\mathrm{FWHM}_{01} / \sqrt{-\mathrm{ER} \ln \Delta p}$ [49], by reducing the ER $[34,50,51]$ and improving the detection fidelity of the dark state $\Delta p$ [52], the resolution can further reach $3.1 \mathrm{~nm}$ under an objective with $\mathrm{NA}=0.6$.

Together with the advantage of nanosecond imaging time, our work opens up new opportunities in the study of single-particle dynamics, many-body correlation [53], 
and even two-body collision dynamics [54] in cold atomic systems. For example, long-range interactions based on Rydberg atoms and ions could induce two-particle entanglement in position and momentum even when they are far apart (with site addressability), and applying fast and high-resolution imaging of each particle allows direct measurement of both two-particle position correlation and particle dynamics, with no need for any additional mapping or time-of-flight operation [53]. Our method can even be used to measure short-range correlations with contact interactions (e.g., the spin-exchange interaction $[3,53])$, as long as the two interacting atoms are distinguishable particles (e.g., different species or spins). We can first coherently transfer a spin-up (or spin-down) state from the $S$ to the $D$ level to store the spin, and then apply the GSD sequence to measure the position of the spin-down (or spin-up) state, while using different wavelengths to image different species.

In conclusion, we have presented a nanosecond-scale super-resolved imaging method to detect a single trapped ion and its dynamics in two dimensions, achieving a spatial-time resolution to $175 \mathrm{~nm}$ at $7 \mu$ s and $373 \mathrm{~nm}$ at $50 \mathrm{~ns}$, and the resolution can be improved further using higher-NA objectives. The method is general and can be used for both charged and neutral cold atoms. It can also be developed further to detect many-body correlations using multiple GSD spots. Furthermore, the GSD method on a cold atom system can be extended in the future to three dimensions, like classical GSD [12], thereby further enhancing its potential.

Note added.-During preparation of the manuscript, we became aware of recent work on optical superresolution sensing of a trapped ion's wave-packet size [55], which uses the ground-state depletion technique to detect the size of the motion wave packet of a trapped $\mathrm{Ca}^{+}$ion.

This work was supported by the National Key Research and Development Program of China (Grant Nos. 2017YFA0304100 and 2016YFA0302700), the National Natural Science Foundation of China (Grants Nos. 11821404, 11874343, 11774335, and 11734015), the Key Research Program of Frontier Sciences, CAS (Grant No. QYZDY-SSW-SLH003), the Science Foundation of the CAS (Grant No. ZDRW-XH-2019-1), the Fundamental Research Funds for the Central Universities (Grant Nos. WK2470000026, WK2470000027, and WK2470000028), the Anhui Initiative in Quantum Information Technologies (Grant Nos. AHY020100 and AHY070000), and the National Program for Support of Topnotch Young Professionals (Grant No. BB2470000005).

Z.-H.Q. and J.-M.C. contributed equally to this work.

* jmcui@ustc.edu.cn

$\dagger$ hyf@ustc.edu.cn
¥ cfli@ustc.edu.cn

[1] W. S. Bakr, J. I. Gillen, A. Peng, S. Fölling, and M. Greiner, Nature 462, 74 (2009).

[2] E. Haller, J. Hudson, A. Kelly, D. A. Cotta, B. Peaudecerf, G. D. Bruce, and S. Kuhr, Nat. Phys. 11, 738 (2015).

[3] A. M. Kaufman, B. J. Lester, M. Foss-Feig, M. L. Wall, A. M. Rey, and C. A. Regal, Nature 527, 208 (2015).

[4] H. Levine, A. Keesling, G. Semeghini, A. Omran, T. T. Wang, S. Ebadi, H. Bernien, M. Greiner, V. Vuletić, H. Pichler, and M. D. Lukin, Phys. Rev. Lett. 123, 170503 (2019).

[5] H. Nägerl, D. Leibfried, F. Schmidt-Kaler, J. Eschner, and R. Blatt, Opt. Express 3, 89 (1998).

[6] J. D. Wong-Campos, K. G. Johnson, B. Neyenhuis, J. Mizrahi, and C. Monroe, Nat. Photonics 10, 606 (2016).

[7] L. A. Zhukas, M. J. Millican, P. Svihra, A. Nomerotski, and B. B. Blinov, Phys. Rev. A 103, 023105 (2021).

[8] B. O. Leung and K. C. Chou, Appl. Spectrosc. 65, 967 (2011).

[9] R. F. Hamans, M. Parente, and A. Baldi, Nano Lett. 21, 2149 (2021).

[10] M. Sunbul, J. Lackner, A. Martin, D. Englert, B. Hacene, F. Grün, K. Nienhaus, G. U. Nienhaus, and A. Jäschke, Nat. Biotechnol. (2021).

[11] E. Rittweger, K. Y. Han, S. E. Irvine, C. Eggeling, and S. W. Hell, Nat. Photonics 3, 144 (2009).

[12] K. Y. Han, K. I. Willig, E. Rittweger, F. Jelezko, C. Eggeling, and S. W. Hell, Nano Lett. 9, 3323 (2009).

[13] P. C. Maurer, J. R. Maze, P. L. Stanwix, L. Jiang, A. V. Gorshkov, A. A. Zibrov, B. Harke, J. S. Hodges, A. S. Zibrov, A. Yacoby, D. Twitchen, S. W. Hell, R. L. Walsworth, and M. D. Lukin, Nat. Phys. 6, 912 (2010).

[14] R. Kolesov, S. Lasse, C. Rothfuchs, A. D. Wieck, K. Xia, T. Kornher, and J. Wrachtrup, Phys. Rev. Lett. 120, 033903 (2018).

[15] M. J. Biercuk, H. Uys, J. W. Britton, A. P. VanDevender, and J. J. Bollinger, Nat. Nanotechnol. 5, 646 (2010).

[16] V. Blums, M. Piotrowski, M. I. Hussain, B. G. Norton, S. C. Connell, S. Gensemer, M. Lobino, and E. W. Streed, Sci. Adv. 4, eaao4453 (2018).

[17] D. D. Yavuz and N. A. Proite, Phys. Rev. A 76, 041802(R) (2007).

[18] A. V. Gorshkov, L. Jiang, M. Greiner, P. Zoller, and M. D. Lukin, Phys. Rev. Lett. 100, 093005 (2008).

[19] J.-M. Cui, F.-W. Sun, X.-D. Chen, Z.-J. Gong, and G.-C. Guo, Phys. Rev. Lett. 110, 153901 (2013).

[20] R. Tenne, U. Rossman, B. Rephael, Y. Israel, A. Krupinski-Ptaszek, R. Lapkiewicz, Y. Silberberg, and D. Oron, Nat. Photonics 13, 116 (2019).

[21] D. Yang, C. Laflamme, D. V. Vasilyev, M. A. Baranov, and P. Zoller, Phys. Rev. Lett. 120, 133601 (2018).

[22] M. Lacki, M. A. Baranov, H. Pichler, and P. Zoller, Phys. Rev. Lett. 117, 233001 (2016).

[23] Y. Wang, S. Subhankar, P. Bienias, M. Lacki, T.-C. Tsui, M. A. Baranov, A. V. Gorshkov, P. Zoller, J. V. Porto, and S. L. Rolston, Phys. Rev. Lett. 120, 083601 (2018).

[24] W. Ge and M. S. Zubairy, Phys. Rev. A 101, 023403 (2020).

[25] M. Lacki, P. Zoller, and M. A. Baranov, Phys. Rev. A 100, 033610 (2019).

[26] T.-C. Tsui, Y. Wang, S. Subhankar, J. V. Porto, and S. L. Rolston, Phys. Rev. A 101, 041603(R) (2020). 
[27] M. McDonald, J. Trisnadi, K.-X. Yao, and C. Chin, Phys. Rev. X 9, 021001 (2019).

[28] S. Subhankar, Y. Wang, T.-C. Tsui, S. L. Rolston, and J. V. Porto, Phys. Rev. X 9, 021002 (2019).

[29] C. Veit, N. Zuber, O. A. Herrera-Sancho, V. S. V. Anasuri, T. Schmid, F. Meinert, R. Löw, and T. Pfau, Phys. Rev. X 11, 011036 (2021).

[30] B. B. Blinov, D. Leibfried, C. Monroe, and D. J. Wineland, Quantum Inf. Process. 3, 45 (2004).

[31] Z. Wang, L. Luo, K. Thadasina, K. Qian, J. Cui, and Y. Huang, EPJ Tech. Instrum. 3 (2016).

[32] S. Olmschenk, K. C. Younge, D. L. Moehring, D. N. Matsukevich, P. Maunz, and C. Monroe, Phys. Rev. A 76, 052314 (2007).

[33] See Sec. II of the Supplemental Material at [URL will be inserted by publisher] for details of the laser system.

[34] P. Zupancic, P. M. Preiss, R. Ma, A. Lukin, M. E. Tai, M. Rispoli, R. Islam, and M. Greiner, Opt. Express 24, 13881 (2016).

[35] M. Beijersbergen, R. Coerwinkel, M. Kristensen, and J. Woerdman, Opt. Commun. 112, 321 (1994).

[36] S. S. R. Oemrawsingh, J. A. W. van Houwelingen, E. R. Eliel, J. P. Woerdman, E. J. K. Verstegen, J. G. Kloosterboer, and G. W. 't Hooft, Appl. Opt. 43, 688 (2004).

[37] M. Beijersbergen, L. Allen, H. van der Veen, and J. Woerdman, Opt. Commun. 96, 123 (1993).

[38] D. V. Petrov, F. Canal, and L. Torner, Opt. Commun. 143, 265 (1997).

[39] S. Slussarenko, A. Murauski, T. Du, V. Chigrinov, L. Marrucci, and E. Santamato, Opt. Express 19, 4085 (2011).

[40] V. D'Ambrosio, N. Spagnolo, L. D. Re, S. Slussarenko, Y. Li, L. C. Kwek, L. Marrucci, S. P. Walborn, L. Aolita, and F. Sciarrino, Nat. Commun. 4 (2013).
[41] L. Yan, P. Gregg, E. Karimi, A. Rubano, L. Marrucci, R. Boyd, and S. Ramachandran, Optica 2, 900 (2015).

[42] Y. Fang-Wei and L. Yong-Ping, Acta Phys. Sinica 52, 328 (2003).

[43] Q. Xiao-Qing, G. Chun-Qing, and L. Yi-Dong, Acta Phys. Sinica 59, 264 (2010).

[44] See Sec. III of the Supplemental Material at [URL will be inserted by publisher] for details of holographic beam reshaping with DMD.

[45] See Sec. VIII of the Supplemental Material [URL will be inserted by publisher].

[46] C. Foot, Atomic Physics (Oxford University Press, Oxford, 2005)

[47] See Sec. VIII of the Supplemental Material at [URL will be inserted by publisher] for a derivation of this limit.

[48] D. Leibfried, R. Blatt, C. Monroe, and D. Wineland, Rev. Mod. Phys. 75, 281 (2003).

[49] See Sec. VIII of the Supplemental Material at [URL will be inserted by publisher] for a derivation of this limit.

[50] L. Yan, P. Gregg, E. Karimi, A. Rubano, L. Marrucci, R. Boyd, and S. Ramachandran, Optica 2 (2015), 10.1364/optica.2.000900.

[51] L. Yan, P. Kristensen, and S. Ramachandran, APL Photonics 4 (2019), 10.1063/1.5045233.

[52] R. Noek, G. Vrijsen, D. Gaultney, E. Mount, T. Kim, P. Maunz, and J. Kim, Opt. Lett. 38, 4735 (2013).

[53] A. Bergschneider, V. M. Klinkhamer, J. H. Becher, R. Klemt, L. Palm, G. Zürn, S. Jochim, and P. M. Preiss, Nat. Phys. 15, 640 (2019).

[54] Q. Guan, V. Klinkhamer, R. Klemt, J. H. Becher, A. Bergschneider, P. M. Preiss, S. Jochim, and D. Blume, Phys. Rev. Lett. 122, 083401 (2019).

[55] M. Drechsler, S. Wolf, C. T. Schmiegelow, and F. Schmidt-Kaler, Phys. Rev. Lett. 127, 143602 (2021). 


\title{
Supplemental Material: Super-resolved imaging of a single cold atom on a nanosecond timescale
}

\author{
Zhong-Hua Qian, ${ }^{1,2}$ Jin-Ming Cui, ${ }^{1,2, *}$ Xi-Wang Luo, ${ }^{3}$ Yong-Xiang Zheng, ${ }^{1,2}$ Yun-Feng \\ Huang, ${ }^{1,2, \dagger}$ Ming-Zhong Ai, ${ }^{1,2}$ Ran He, ${ }^{1,2}$ Chuan-Feng Li, ${ }^{1,2, \ddagger}$ and Guang-Can Guo ${ }^{1,2}$ \\ ${ }^{1}$ CAS Key Laboratory of Quantum Information, \\ University of Science and Technology of China, Hefei 230026, China \\ ${ }^{2}$ CAS Center For Excellence in Quantum Information and Quantum Physics, \\ University of Science and Technology of China, Hefei 230026, China \\ ${ }^{3}$ Department of Physics, The University of Texas at Dallas, \\ Richardson, Texas 75080-3021, USA
}

(Dated: November 23, 2021) 


\section{ION TRAP SETUP}

The ion trap in this experiment is a three-dimensional (3D) needle trap, as shown in Fig. S1. The trap electrodes consist of six tungsten needles [1]. Each needle has a diameter of $0.5 \mathrm{~mm}$, and adjacent needles are $1 \mathrm{~mm}$ apart. Opposite rows of electrodes are perpendicular to each other. The two central electrodes have a tip-to-tip distance of $170 \mu \mathrm{m}$ and are supplied with $22 \mathrm{MHz}$ radio frequency $(\mathrm{RF})$. All electrodes are supplied with a static (DC) voltage ranging from $-5 \mathrm{~V}$ to $5 \mathrm{~V}$ to modify the ion's position. This 3D trap is suitable for trapping a single ${ }^{171} \mathrm{Yb}^{+}$ion. To provide a quantum axis for the spin, a $9.7 \mathrm{G}$ magnetic field along the $z$ axis is needed.

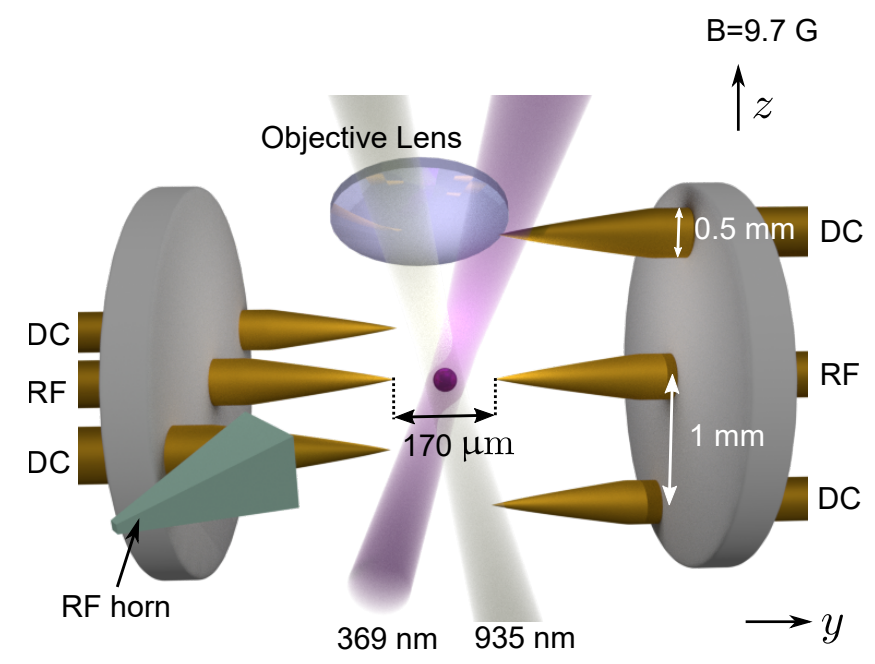

FIG. S1. Electrode configuration of the needle traps (not to scale).

\section{LASER CONFIGURATION FOR ${ }^{171} \mathrm{Yb}^{+}$}

The state operation on ${ }^{171} \mathrm{Yb}^{+}$involves Doppler cooling, pumping (or initialization), detection, and depletion. The ${ }^{171} \mathrm{Yb}^{+}$ion is Doppler-cooled by laser light whose frequency is $\Delta_{1}=10 \mathrm{MHz}$ red-detuned from the transition ${ }^{2} \mathrm{~S}_{1 / 2}|\mathrm{~F}=1\rangle$ (bright state) to ${ }^{2} \mathrm{P}_{1 / 2}|\mathrm{~F}=0\rangle$ and ${ }^{2} \mathrm{~S}_{1 / 2}|\mathrm{~F}=0\rangle$ (dark state) to ${ }^{2} \mathrm{P}_{1 / 2}|\mathrm{~F}=1\rangle[2]$. A repumping beam (935 $\mathrm{nm}$ ) is used to repump the ion from ${ }^{2} \mathrm{D}_{3 / 2}$ to the ${ }^{3} \mathrm{D}[3 / 2]_{1 / 2}$ state, from which the electron will decay to the ${ }^{2} \mathrm{~S}_{1 / 2}$ state, forming a closed cooling cycle. A $638 \mathrm{~nm}$ laser beam pumps the ion from ${ }^{2} \mathrm{~F}_{7 / 2}$ to ${ }^{1} \mathrm{D}[5 / 2]_{5 / 2}$ so that it will decay back to the cooling cycle. Initialization to the 


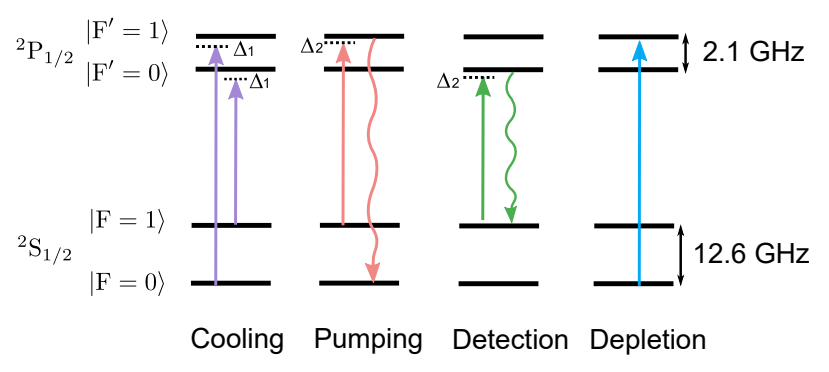

FIG. S2. ${ }^{171} \mathrm{Yb}^{+}$energy level diagram for Doppler cooling, pumping, detecting, and depletion.

ground state is realized with pumping light that is $\Delta_{2}=3 \mathrm{MHz}$ red-detuned from the transition ${ }^{2} \mathrm{~S}_{1 / 2}|\mathrm{~F}=0\rangle$ to ${ }^{2} \mathrm{P}_{1 / 2}|\mathrm{~F}=1\rangle$. The ion is initialized into the dark state ${ }^{2} \mathrm{~S}_{1 / 2}|\mathrm{~F}=0\rangle$ within tens of microseconds. Detection is achieved by a state-dependent fluorescence technique using laser light of the transition ${ }^{2} \mathrm{~S}_{1 / 2}|\mathrm{~F}=1\rangle$ to ${ }^{2} \mathrm{P}_{1 / 2}|\mathrm{~F}=0\rangle$, which is also $3 \mathrm{MHz}$ red-detuned from resonance. The ion will scatter fluorescence photons only when in the ${ }^{2} \mathrm{~S}_{1 / 2}|\mathrm{~F}=1\rangle$ state (bright state). The ion is depopulated from the dark state into the bright state by the depletion laser, whose wavelength is near-resonant with the transition between ${ }^{2} \mathrm{~S}_{1 / 2}|\mathrm{~F}=0\rangle$ and ${ }^{2} \mathrm{P}_{1 / 2}|\mathrm{~F}=1\rangle$.

The laser configuration is shown in Fig. S3. Laser 1 (wavelength $369.52624 \mathrm{~nm}$ ) provides cooling, pumping, and detection beams. For the cooling laser, an electro-optic modulator (EOM 1) provides a $14.7 \mathrm{GHz}$ sideband. The detection beam and pumping beam share the same path and are switched by turning off/on EOM $2(2.1 \mathrm{GHz})$. These two beams are combined and directed to the ion trap from the side window (see Fig. S4). The depletion beam is directed to the digital micromirror device (DMD) path and is then is reshaped into a donut beam (for the setup, see Fig. S5), which will be described later. Laser 2 (wavelength $369.51945 \mathrm{~nm}$ ) is $14.7 \mathrm{GHz}$ blue-detuned with laser 1. Both lasers are frequency-stabilized within $1 \mathrm{MHz}$ through the PDH locking technique [3] by locking to an ultrastable cavity. Acousto-optic modulators (AOMs) are used to switch beams.

Of the laser beams directed into the side windows (Fig. S4), the Doppler cooling, detection, and pumping lasers are at $45^{\circ}$ to the $y$ axis to ensure that the $k$ vector is not orthogonal to any motion mode. Thus, the ion will be efficiently Doppler-cooled in three axes. The $\mathrm{Yb}$ atoms are ionized by a two-photon transition with $369 \mathrm{~nm}$ and $399 \mathrm{~nm}$ ionization beams with powers of $200 \mu \mathrm{W}$ and $500 \mu \mathrm{W}$, respectively.

The depletion beam is reshaped into a donut beam and is then incident into objective 1 


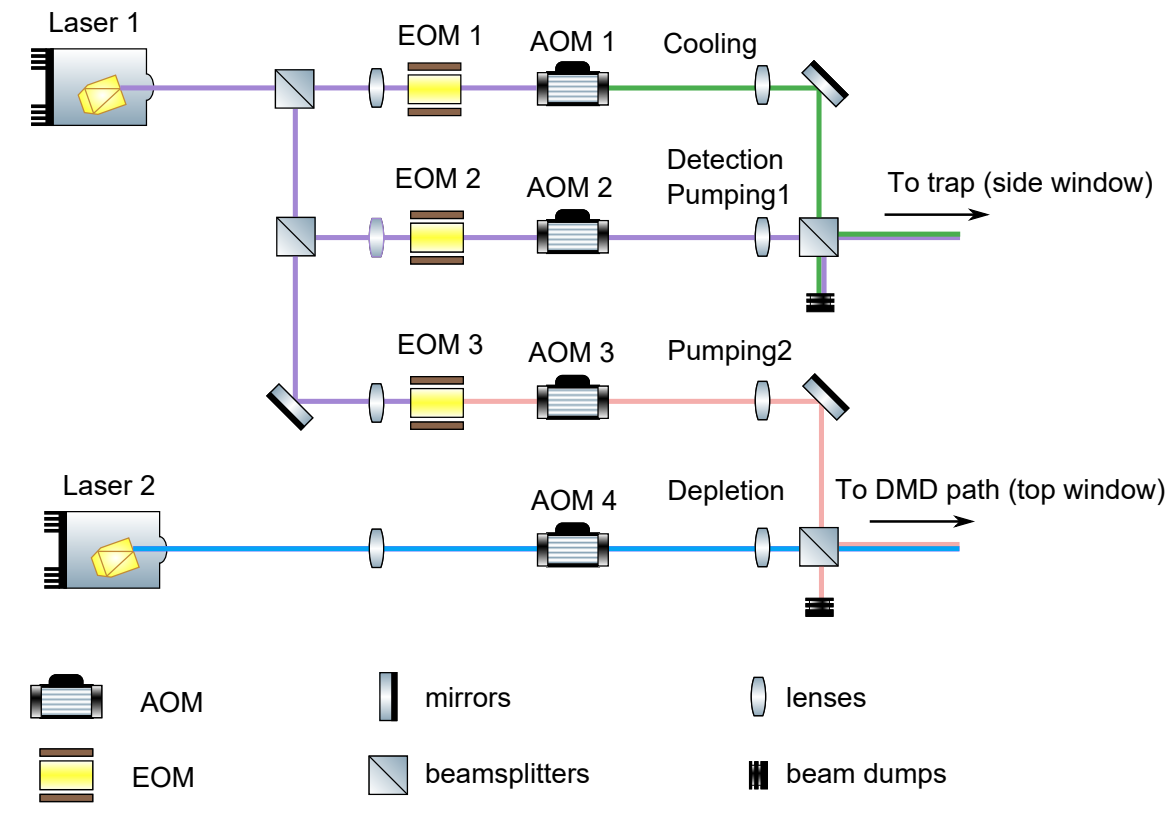

FIG. S3. Laser configuration for Doppler cooling, pumping, detecting, and depletion.

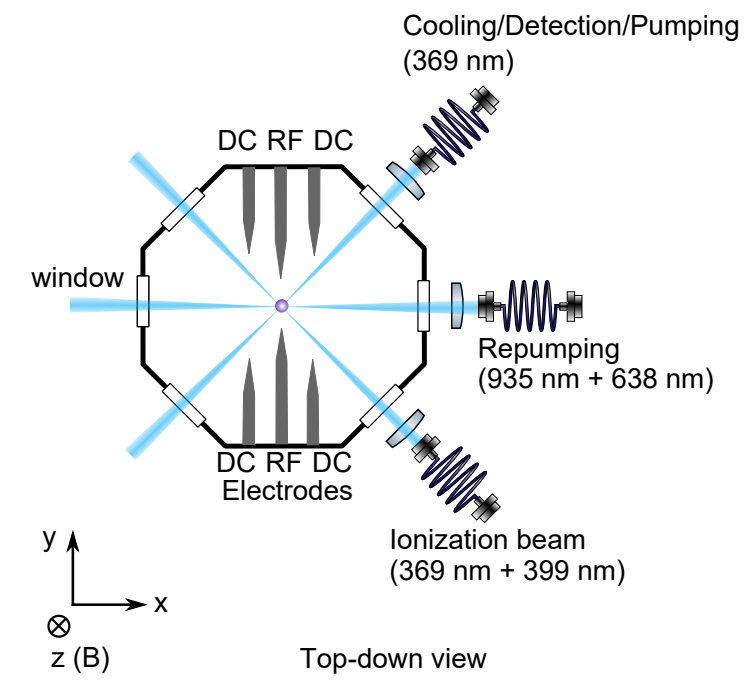

FIG. S4. Top view of the laser configuration of the 3D needle trap. The waists of the cooling, repumping, and ionization beams are $20 \mu \mathrm{m}, 60 \mu \mathrm{m}$, and $30 \mu \mathrm{m}$, respectively.

(NA $=0.1)$. The beam shaping setup is illustrated in Fig. S5. The objectives obj 1 and obj 2 $(\mathrm{NA}=0.1)$ are both used to image the ion. The imaging system of obj 1 has a magnification of 30. The main function of obj 2 is to calibrate the position of the depletion beam to overlap with the ion. The calibration procedure is as follows. First, we use a laser beam focused 


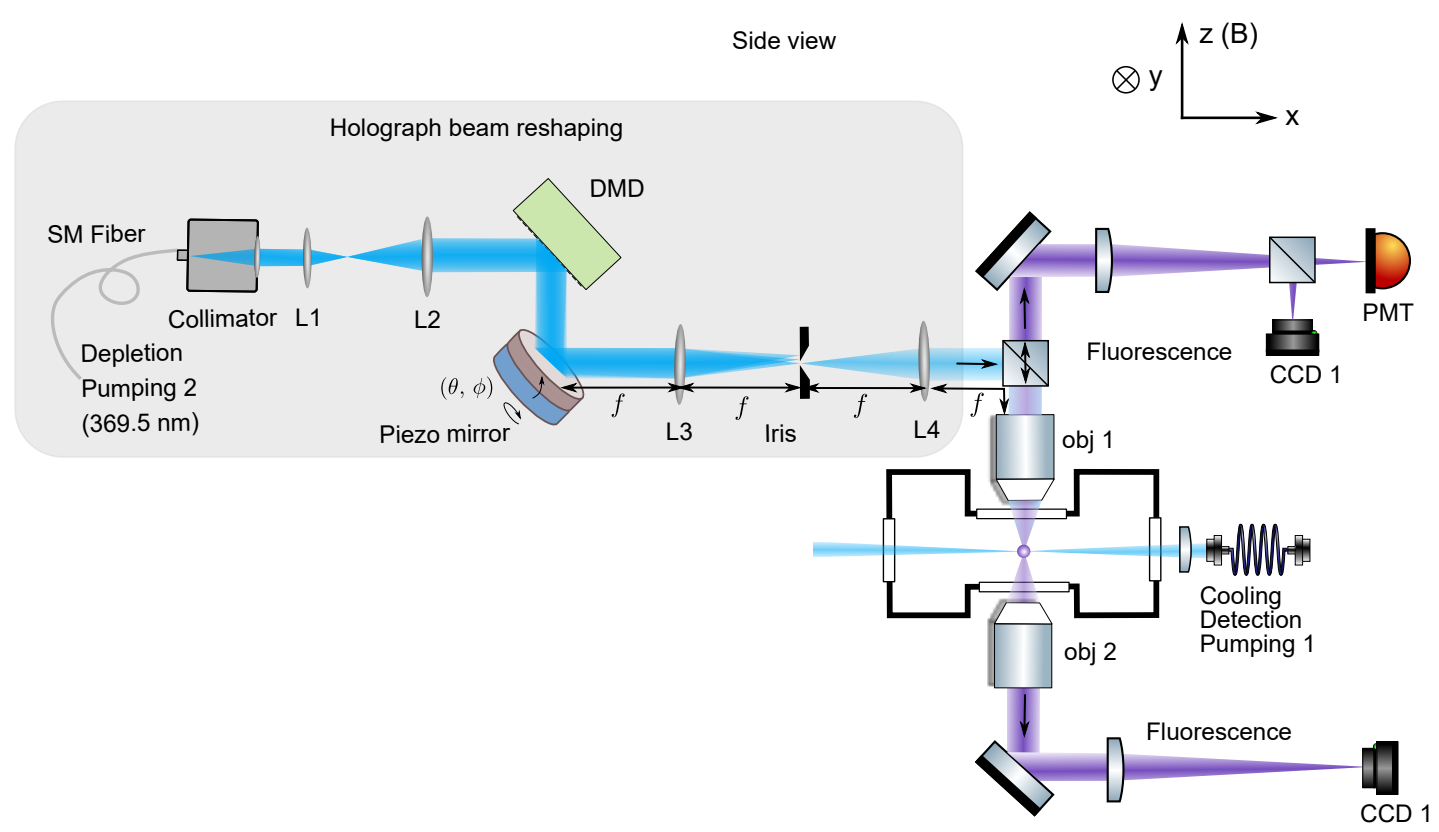

FIG. S5. Side view of the laser configuration of the needle trap. The setup in the gray box is used to reshape the Gaussian beam into a donut beam. The first-order diffraction beam is phase-modulated and filtered by an iris. The objective obj 1 serves for state detection and focusing of the donut beam, while obj 2 is used to precisely calibrate the position of the donut spot.

by obj 2 to simulate the trajectory of the ion's fluorescence. We adjust obj 2's lateral and focal position to ensure that the simulation beam's image coincides with the ion's image on CCD 1. Second, we use CCD 2 to locate the ion's position. We then fine-tune the direction of the depletion beam to make their images overlap on CCD 2. Third, we use the ion as an intensity probe to tune the depletion beam's position more precisely. The depletion light and detection light are turned on at the same time, resulting in Doppler cooling cycles. The intensity of the depletion light is characterized in terms of photon counts per second. We carefully tune the path of the depletion laser to ensure that the ion at its maximum emission rate.

\section{HOLOGRAPHIC BEAM RESHAPING WITH DMD}

In ground-state depletion (GSD) super-resolution schemes, the first-order LaguerreGaussian mode $\left(\mathrm{LG}_{0}^{1}, p=0, l=1\right)$ is often used as a donut beam. We use a holographic beam reshaping method to produce the Laguerre-Gaussian mode. The main advantage of 
(a)

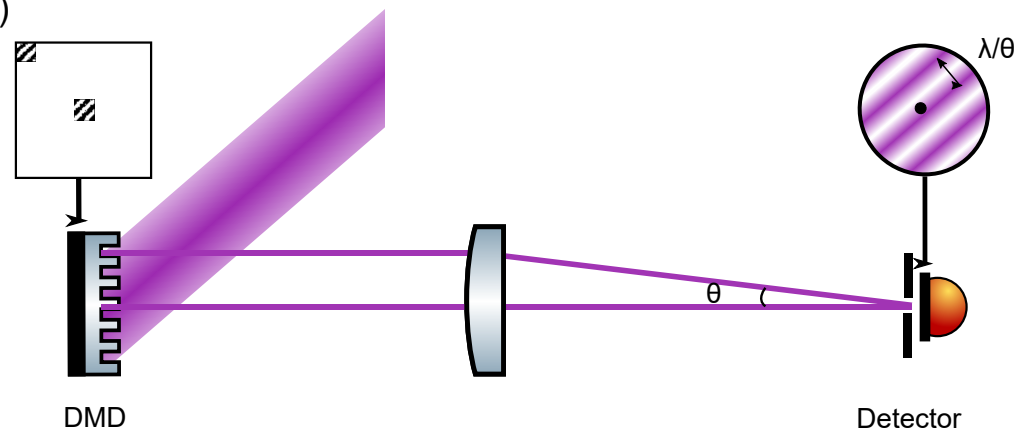

(b)

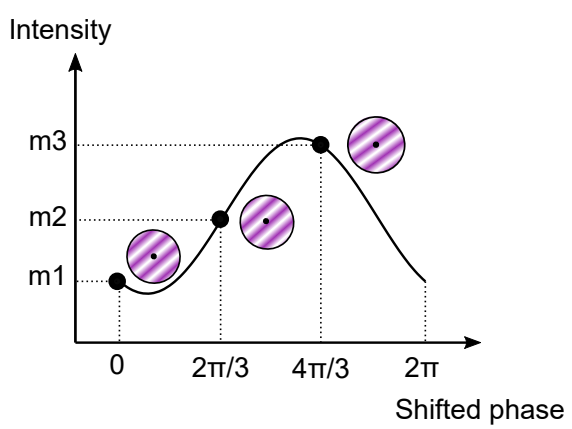

(c)

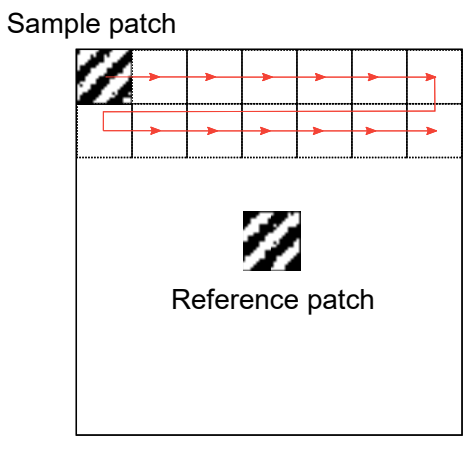

FIG. S6. Concept of aberration correction using a DMD. (a) Interference of the beams diffracted by the sample patch and reference patch. The DMD is illuminated by a collimated laser. The laser is then focused by a lens. The first-order diffraction beams interfere on the image plane. The detector measures the intensity at the central position (black dot) of the interference pattern. The detector can be an ion, CCD, or PMT. (b) Three-step phase-shift method to recover the phase. The phase of the sample patch is shifted to $0,2 \pi / 3$, and $4 \pi / 3$, which results in three intensity values $m_{0}$, $m_{1}$, and $m_{2}$. The initial phase $\phi$ of the sample patch is calculated from these. (c) Acquisition of the phase map by scanning the sample patch while keeping the reference patch fixed. The default phase of the reference patch is set to zero.

this method lies in its ability to perform aberration sensing and correction [4, 5].

The holograph beam reshaping setup is illustrated in Fig. S5 (gray box). The collimated depletion light is expanded 2.7 times by L1 $\left(f_{1}=75 \mathrm{~mm}\right)$ and L2 $\left(f_{2}=200 \mathrm{~mm}\right)$. The $27 \mathrm{~mm}$ diameter of the incident beam is large enough to cover the whole DMD area $(20.7 \times$ $11.7 \mathrm{~mm}^{2}$ ). A holograph pattern on the DMD diffracts the beam into multiple orders. The DMD area that we use is $660 \times 660$ pixels. The period of the holograph grating is 10 pixels. The first-order beam is phase-modulated and is selected by an iris. The diffraction 
(a) Original phase map

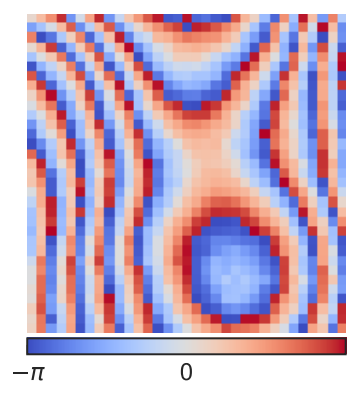

(b) Unwrapped phase map

(c) Interpolated phase map

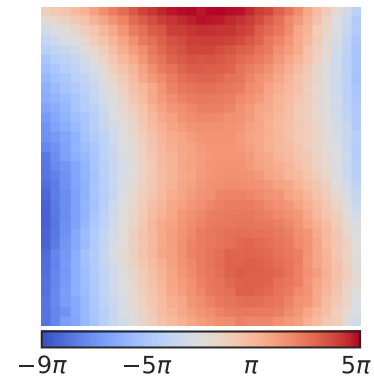

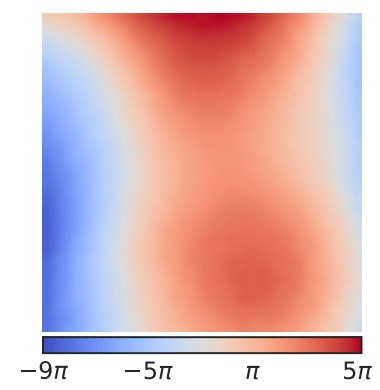

FIG. S7. Measured phase map. (a) Original phase map, whose values lie in the range $[-\pi, \pi]$. (b) Unwrapped phase map. The phase aberration ranges over $14 \pi$, corresponding to $7 \lambda$. (c) Interpolation of (b) to each pixel.

efficiency is $\sim 5 \%$. The piezo mirror (PI, S-330.2SH) is used to scan the donut spot on the ion's plane in two dimensions; the scanning area $\left(50 \times 50 \mu \mathrm{m}^{2}\right)$ is large enough to cover the ion's trajectory. The scanning scale between the tip/tilt angle and the displacement on the ion's plane is $25 \mathrm{~nm} / \mu \mathrm{rad}$, which is calibrated with the obj 2 imaging system. The beam at the piezo mirror is conveyed to the entrance of obj 1 through a 4 -f system $(f=300 \mathrm{~mm})$. The entrance pupil diameter of the spot is $7.1 \mathrm{~mm}$.

To generate the donut beam, the first step is to measure the aberration of the optical system via the DMD (ViALUX v9501). A DMD is a binary spatial light modulator, which in this case is made of $1920 \times 1080$ aluminum micromirrors. Each micromirror has a width of $10.8 \mu \mathrm{m}$. It flips only $+12^{\circ}$ or $-12^{\circ}$ along a hinge corresponding to on or off states. The main idea of phase calibration is to measure the phase of a certain region (sample patch, $20 \times 20$ pixels) relative to the phase of the center region (reference patch, $20 \times 20$ pixels) through a phase-shifting technique $[6,7]$. The procedure for aberration correction is as follows (see Fig. S6). First, we turn on the micromirrors of the sample patch and reference patch. The phase of the reference patch is a global phase and so can be regarded as zero. Two beams diffracted by holograph patterns will interfere on the ion's plane. The ion serves as a detector of interference pattern intensity. Shifting the phase of the sample patch will lead to a shift of the interference pattern. The relative phase can thus be measured via a three-step phase shift technique. We change the sample patch while fixing the reference patch and repeat the above steps until the whole phase map has been measured. The entire process takes about 20 min. 
The measured phase map is shown in Fig. S7(a) and ranges from $-\pi$ to $\pi$. It needs to be unwrapped to give a continuous phase map. The unwrapped phase map [Fig. S7(b)] ranges from $-9 \pi$ to $5 \pi$, indicating that the peak-to-valley of aberration is $7 \lambda$. The unwrapped phase is further interpolated to each pixel to recover the tip/tilt terms of each patch. Thus, diffracted beams focused by obj 1 can overlap well on the ion's plane, which ensures higher accuracy of phase sensing. Finally, we obtain the smooth phase map [see Fig. S7(c)].

The aberration measurement procedure is followed by beam reshaping. To generate the Laguerre-Gaussian mode beam, phase and amplitude modulation are implemented. The target phase is the sum of the aberration phase maps in Fig. S7(c) and the ideal Laguerre-Gaussian phase map [Fig. S8(b)]. For amplitude modulation, the ratio of "on" states in the computer-generated holograph pattern is proportional to the amplitude profile of the Laguerre-Gaussian mode [Fig. S8(a)]. Finally, the phase map and amplitude map are binarized into a holograph pattern using the Lee method [8]. The pattern is then projected on the DMD. With this method, a Gaussian beam with high mode purity can also be achieved.

The piezo mirror is scanned in two dimensions to measure the intensity profiles of the donut spot and Gaussian spot. The power of the depletion light here is $125 \mathrm{pW}$. The results are shown in Fig. S9. The FWHMs of the reshaped Gaussian and donut spots are $2.34 \mu \mathrm{m}$ and $1.4 \mu \mathrm{m}$, respectively. The residual intensity of the central dark area is $3.8 \%$ of the maximum intensity of the donut spot, which verifies the high mode purity of the donut spot.

\section{SEQUENCE OF SUPER-RESOLVED IMAGING}

The sequence of super-resolved imaging is illustrated in Fig. S10. The whole sequence can

be divided into two layers of nested loops. In the outer layer, the piezo mirror is varied to scan the donut spot over a series of positions $\left\{\left(x_{i}, y_{j}\right) \mid i, j=0,1,2, \ldots, N\right\}$, where $N=20$ in this work. At each position $\left(x_{i}, y_{j}\right)$, we run the sequence in the second layer 100 times. The ion is first Doppler-cooled for $1 \mathrm{~ms}$. Then, the global pumping beam initializes the ion into a dark state within $50 \mu \mathrm{s}$. Next, the pulsed donut spot depopulates the ion into a bright state. Finally, the ion's state is read out by the detection laser. This inner layer cycles 100 times to produce the dark-state probability. The position is then changed to the next point, and the inner sequences are repeated. 
(a) LG mode ( $p=0,1=1)$ amplitude profile

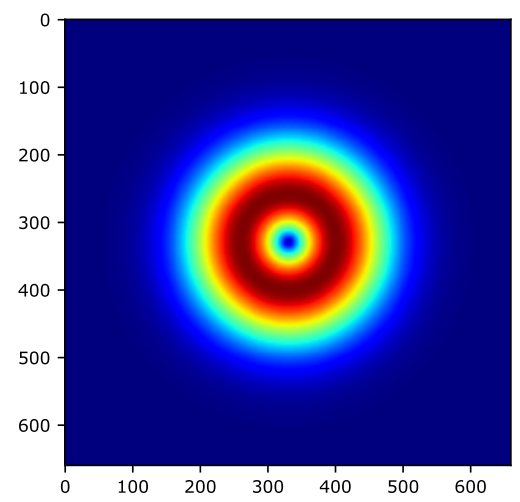

(c) LG mode ( $p=0, \mathrm{l}=1)$ biniarized pattern

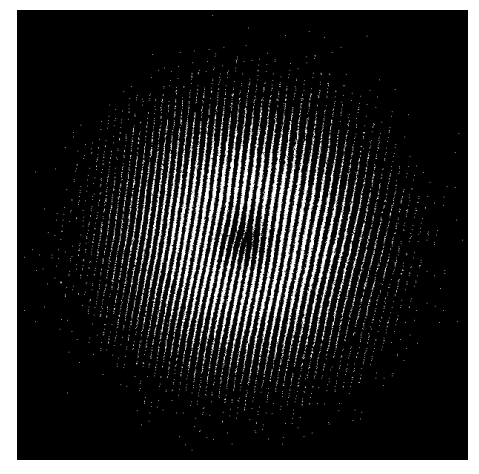

(b) LG mode $(p=0, I=1)$ phase profile

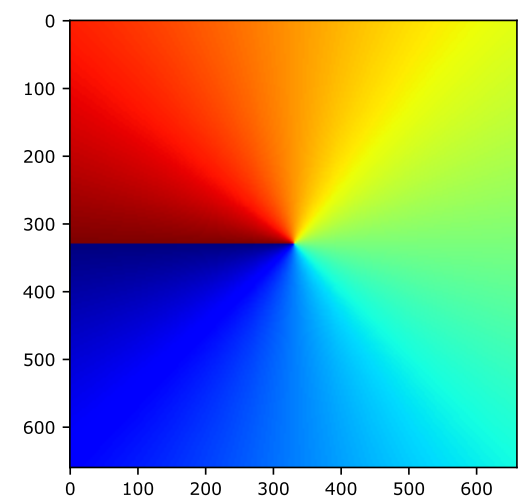

(d)

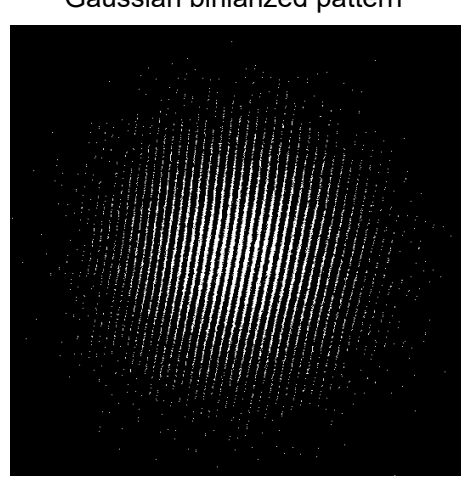

FIG. S8. Beam reshaping holographic patterns. (a) and(b) Phase and amplitude profiles of the donut beam. (c) and (d) Holographic patterns of donut modes and Gaussian modes on DMD.

In our current study, we just used a global initialization beam to initialize the ion, since there are no other ions around it. This is a simplified scheme of GSD. In the case of multiple atoms with small spacing, to resolve a cluster of atoms with subdiffraction resolution, instead of the global Gaussian beam, it is necessary to use a small Gaussian spot, as in Fig. 2(a) of the main paper, to initialize the emitter into the ground state locally, in the standard GSD microscopy scheme. It is easy to achieve this requirement with our setup by projecting Gaussian and donut holographic patterns on the DMD in sequence. The lasers for initialization and depletion (turning on and off are controlled by AOM 3 and AOM 4, respectively, in Fig. S3) are combined and guided to the DMD beam reshaping setup. In the initialization process, we load the holograph pattern for a Gaussian spot on the DMD and then switch AOM 3 on; after switching AOM 3 off to finish the initialization, we then load the holograph pattern for the Gaussian spot on the DMD. In the depletion process, we switch AOM 4 on for the duration time for depletion. The holograph patterns have 
(a)

(c)
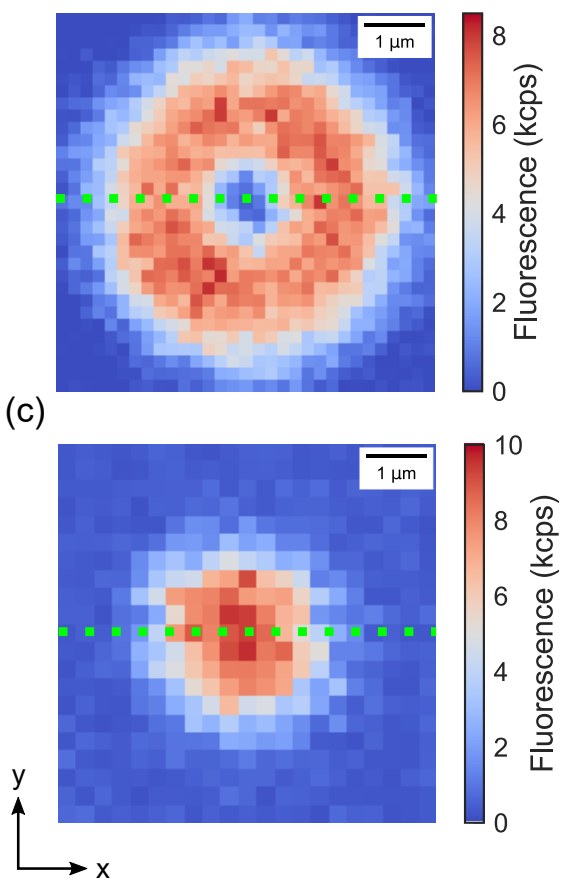

(b)

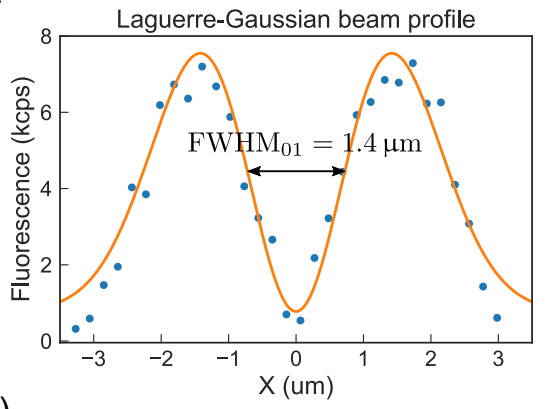

(d)

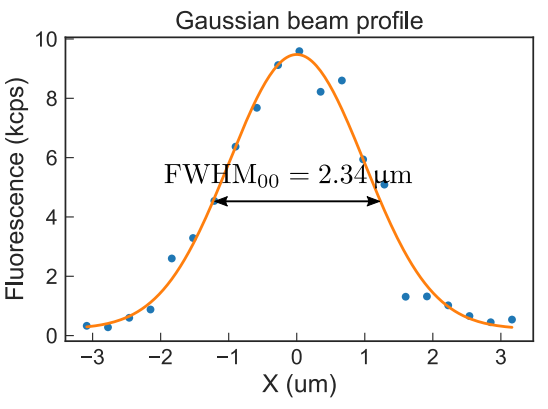

FIG. S9. Reshaped Gaussian and donut spots. The intensity is represented by the number of fluorescence photons measured per second (kcps = kilocounts per second). Background noise has been eliminated. (a) and (b) Donut spot ( $\mathrm{LG}_{0}^{1}$ mode). The minimum intensity in the center is $3.8 \%$ of the maximum intensity. The FWHM of the central valley is $1.4 \mu \mathrm{m}$. (c) and (d) Gaussian beam with FWHM $2.34 \mu \mathrm{m}$. The data for the curves in (b) and (d) is extracted from the green dashed lines in (a) and (c).

been pre-programmed into the FPGA board of the DMD, and so we can rapidly switching these patterns by an electrical trigger. The maximum switching rate is $17857 \mathrm{~Hz}$, which corresponds to a switch time of $6 \mu \mathrm{s}$. Because turning the lasers on and off is controlled by AOMs, the depletion process can still be as fast as nanoseconds, and only the holograph pattern switching process causes a time delay. To shorten the holograph pattern switching time, we can use two DMD beam reshaping setups to compensate and generate the Gaussian and donut spots individually. 


\section{Super-resolved imaging sequence}

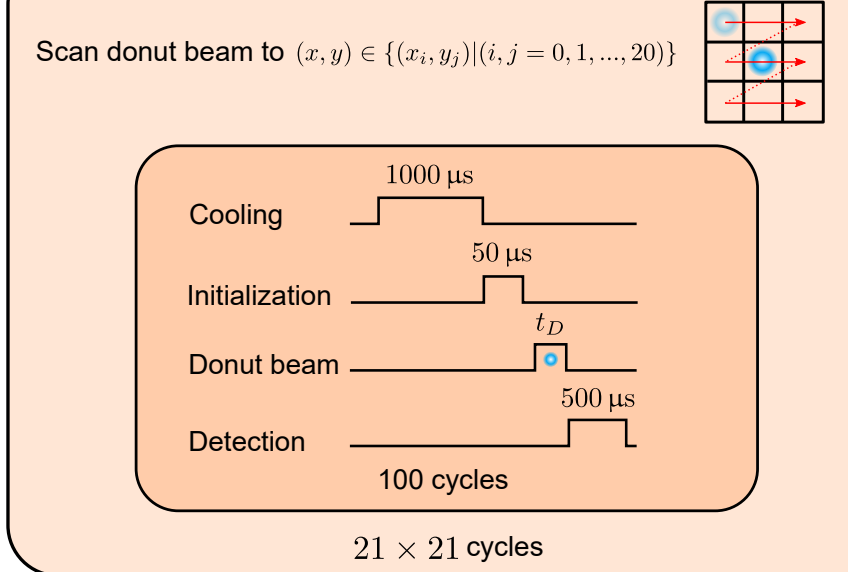

FIG. S10. Sequence of super-resolved imaging. It comprises two layers of nested loops. At each position of the donut spot, the inner layer of the sequence is cycled 100 times.

\section{MOTION FREQUENCY STABILIZATION}

The ion's frequencies of motion on the $x, y, z$ axes are $804 \mathrm{kHz}, 1.36 \mathrm{MHz}$ and $847 \mathrm{kHz}$ respectively, where the $y$ axis is defined as lying along the central RF electrodes. To resolve the ion's dynamics, we drive its motion on the $y$ axis with a resonant RF voltage. Therefore, its motion frequency is required to remain stable under driving. To stabilize this frequency, active feedback is designed and implemented [9]. The motion frequency is proportional to the trapping voltage divided by the RF frequency. The drift of the trapping voltage due to thermal fluctuations and amplifier noise is the main reason for destabilization of the motion frequency. To stabilize this frequency, feedback to the voltage on the trap electrodes is implemented. The scheme is illustrated in Fig. S11. A high voltage is first sampled with a capacitor divider network comprising a $0.2 \mathrm{pF}$ and a $20 \mathrm{pF}$ capacitor. The sampling AC voltage is $1 \%$ of the trapping voltage. This sampling voltage then passes through a rectifier and is transformed into a DC voltage. This signal is acquired with an analog-to-digital converter (ADC). The ADC that we use is a digital multimeter (Rigol DM3068). It has a high accuracy of 6.5 bits and a minimum resolved voltage of $1 \mu \mathrm{V}$. The digital signal is transmitted to a computer, which serves as a servo controller. It runs a proportionalintegral-derivative (PID) algorithm with a set point $0.39 \mathrm{~V}$ and outputs a DC signal through 


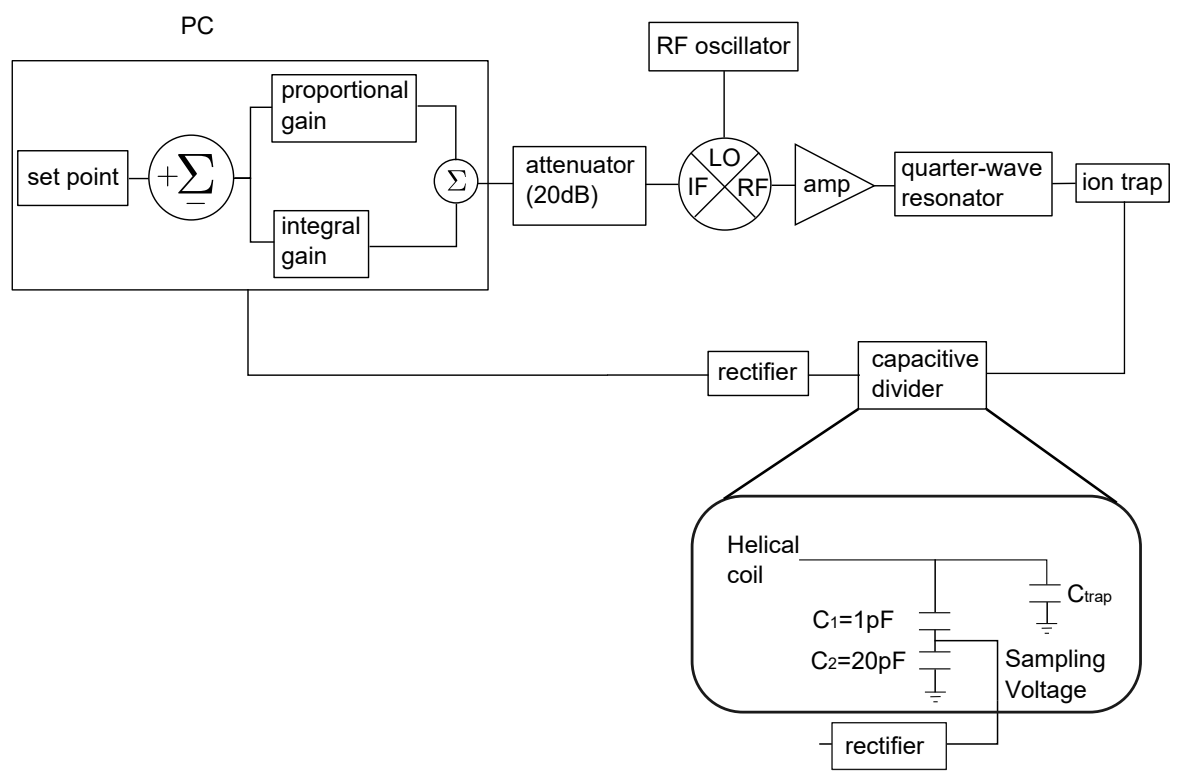

FIG. S11. Scheme of motion frequency feedback.

a 24-bit digital-to-analog converter (DAC). The signal is attenuated by $20 \mathrm{~dB}$ to make the output voltage large enough. Thus, we can make the greatest use of the high accuracy of the DAC. The attenuated signal is mixed with a local oscillator (LO) signal generated by an RF source $(22 \mathrm{MHz},-25 \mathrm{dBm})$. The output signal is then amplified by $51 \mathrm{~dB}$ and finally transmitted into the trap via a quarter-wave resonator.

The result is shown in Fig. S12. The standard error of the relative voltage $\delta V / V$ is suppressed to within $88 \mathrm{ppm}$. The occasional spikes may result from the low feedback speed $(2 \mathrm{~Hz})$, which is limited by the sampling rate of the ADC. The drift of the motion frequency fluctuates within $0.5 \mathrm{kHz}$ within an hour. In the two-dimensional (2D) motion detection scheme, the RF driving time is $73.5 \mu \mathrm{s}$, which means that the fluctuation of the $y$-mode motion frequency should be far less than $7 \mathrm{kHz}$. This feedback system meets our requirements.

\section{SEQUENCE OF 2D MOTION DETECTION}

The super-resolved technique is applied to resolve the ion's dynamics in two dimensions. Here we drive the ion's $y$-mode motion with a resonant RF signal (1.36 MHz).

The sequence is as shown in Fig. S13. It is divided into three layers of nested loops. In 


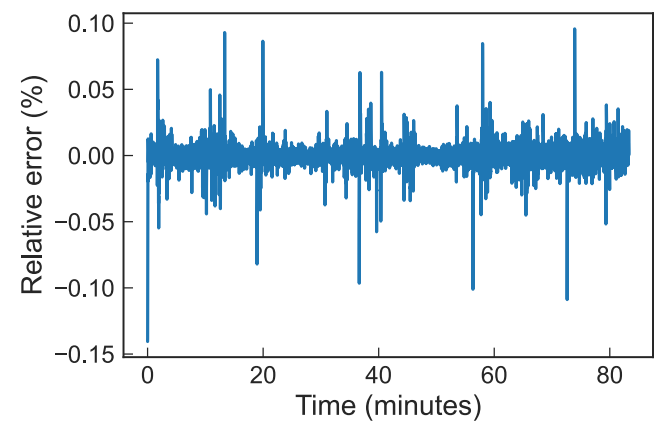

FIG. S12. Drift of the sampling voltage. The relative voltage error $\delta V / V$ is defined as the difference between output voltage and the set point divided by the set point.

the first layer, we set the delay time $t$ to a series of values to observe the ion's motion at different oscillation phases. We sample 10 points within a motion period ( $T=735 \mathrm{~ns})$. In the second layer, similar to the super-resolved sequence, we scan the donut spot to a series of positions $\left(x_{i}, y_{j}\right)$. The scanning area is $1.25 \times 1.25 \mu \mathrm{m}^{2}$, with $10 \times 10$ points. In the last layer, we drive the ion for 100 periods with an RF signal. We then stop driving and wait for a time $t$ followed by a $50 \mathrm{~ns}$ depletion process with the donut spot. $I_{\max } / I_{\text {sat }}$ is fixed at 450. This third layer is cycled 100 times to obtain the dark-state probability.

\section{DEPLETION PROCESS SIMULATION AND CONTROL}

The depletion interaction can be modeled by an optical pumping process, as shown in Fig. S14(a). The probability evolution of the dark state is described by the master equation

$$
\partial_{t} \rho(t)=-\frac{i}{\hbar}[H, \rho(t)]+\sum_{n} \frac{1}{2}\left[2 C_{n} \rho(t) C_{n}^{+}-\rho(t) C_{n}^{+} C_{n}-C_{n}^{+} C_{n} \rho(t)\right],
$$

where $H=\frac{1}{2} \Omega|1\rangle\langle 2|+$ H.c., $C_{1}=-\sqrt{\frac{1}{3} \Gamma}|1\rangle\left\langle 2\left|, C_{2}=\sqrt{\frac{1}{3} \Gamma}\right| 3\right\rangle\left\langle 2\left|, C_{3}=\sqrt{0 \times \Gamma}\right| 4\right\rangle\langle 2|$, and $C_{5}=-\sqrt{\frac{1}{3} \Gamma}|5\rangle\langle 2|$ are the collapse operators representing the decays of the excited state $|2\rangle$ to $|1\rangle,|3\rangle,|4\rangle$, and $|5\rangle . \Gamma=2 \pi \times 19.6 \mathrm{MHz}$ is the spontaneous emission rate of $|2\rangle$. We simulate the probability evolution of the dark state with QuTiP [10]. We use the saturation parameter $s$ to characterize the light intensity:

$$
s=\frac{I}{I_{\mathrm{sat}}}=\frac{2 \Omega^{2}}{\Gamma^{2}}
$$




\section{Motion detection sequence}

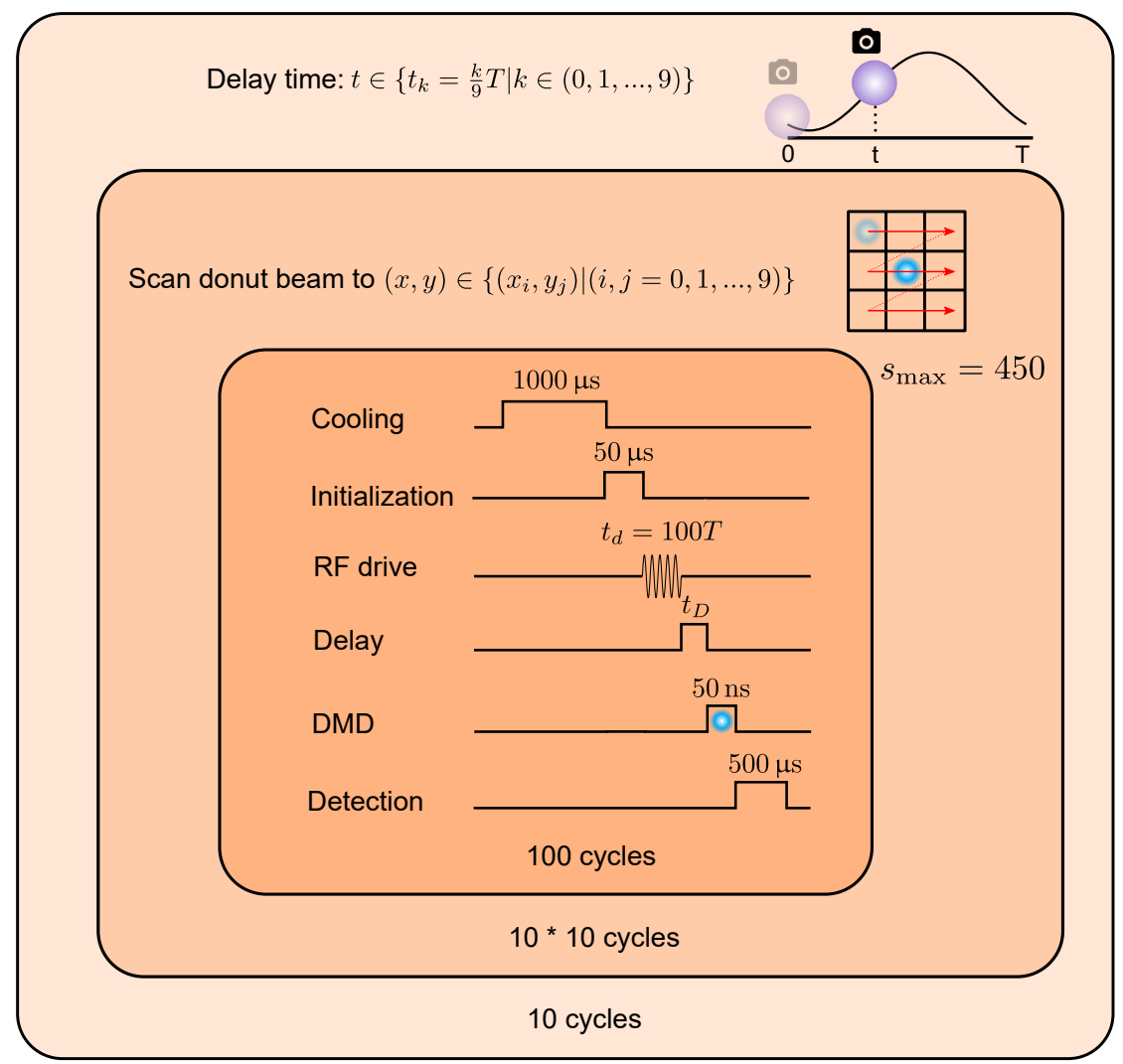

FIG. S13. Motion detection sequence. It comprises three layers of nested loops. In the first layer, the delay time $t$ is set to a series of values, meaning that the ion is observed at different motion phases. In the second layer, the piezo mirror's angle is set to scan the donut spot. In the third layer, the ion is driven by a pulsed RF signal, and the depletion beam then takes a fast snapshot of the moving ion.

where $I_{\text {sat }}$ is the saturation intensity and $\Omega$ is the Rabi frequency.

The result is shown in Fig. S14. For $s=100$, it takes 50 ns for the probability of the dark state to drop below 0.05. The depletion time will not decrease linearly, even for larger values of the saturation parameter. Considering that too short a donut pulse would lead to inefficient depopulation of the dark state, we set the depletion time to 50 ns. This pulse duration is far shorter than the motion period (735 ns), which is sufficient to resolve the ion's dynamics.

In an experiment, the pulse duration is also limited by the rise time of the AOMs, as illustrated in Fig. S15. We observed a rise time of 23 ns and a fall time of 25 ns from $20 \%$ 
(a)

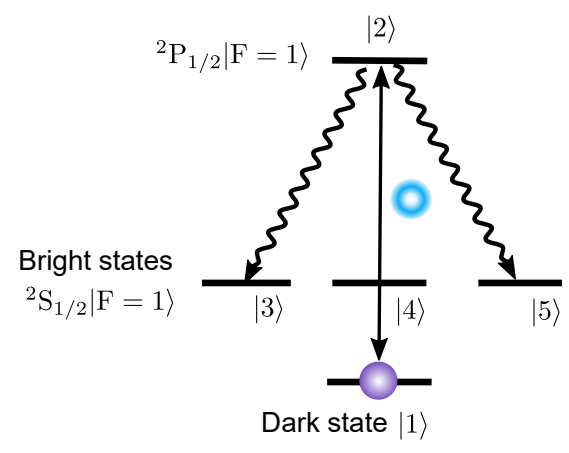

(b)

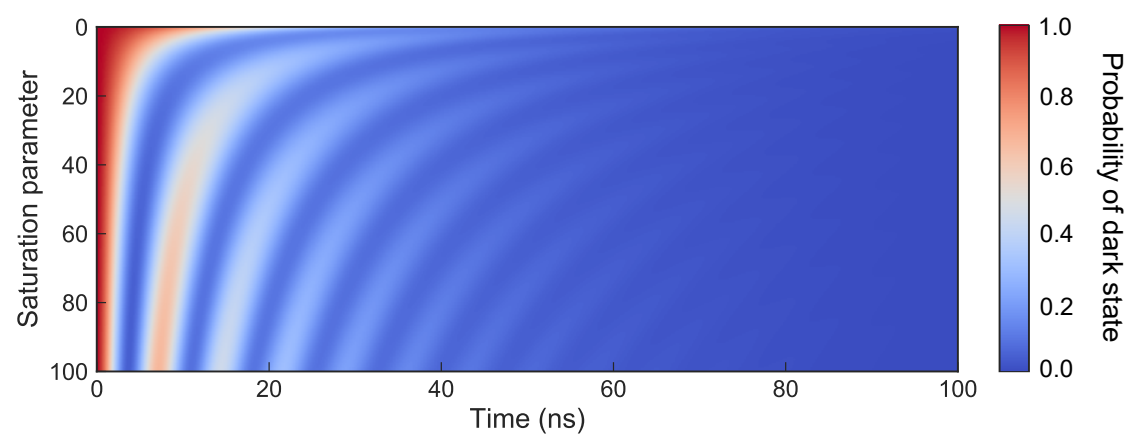

FIG. S14. Numerical simulation of depletion process under different intensities. (a) Model of three-level system under a pulsed depletion beam. (b) Probability of dark state vs time with different intensities. The intensity is characterized by the saturation parameter. As the saturation parameter increases, the speed with which the probability approaches zero becomes much slower.

to $100 \%$ intensity. The modulation efficiency will drop dramatically when the pulse time decreases below 50 ns.

Figure S16 depicts the ion's trajectory of motion under driving voltages of $V_{\mathrm{pp}}=38$, $82 \mu \mathrm{V}, 122 \mu \mathrm{V}$, and $216 \mu \mathrm{V}$. The ion's position from 2D Gaussian fitting is indicated by a blue cross.

We also measured the ion's motion in two periods (Fig. S17) The motion period is fitted as $723.2 \pm 9.9 \mathrm{~ns}$. The relative error is $1.6 \%$.

\section{IMAGING RESOLUTION}

In this section, we will derive a formula for the resolution. The ion is optically pumped to the bright state by a donut spot with duration $t_{D}$. In this regime, the duration is much longer than the natural lifetime $\left(t_{D} \gg 1 / \Gamma\right)$. The dark-state probability $p_{0}$ can be modeled 
(a)

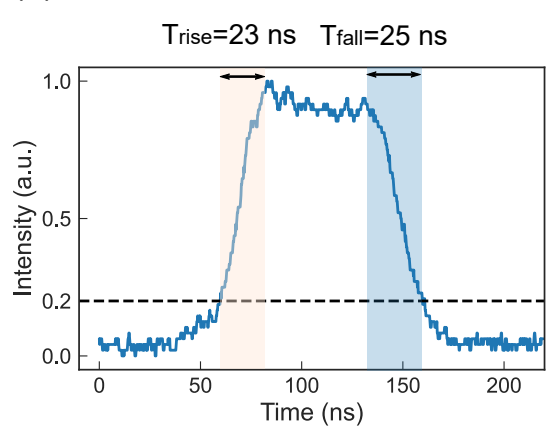

(b)

Turn AOM on $50 \mathrm{~ns}$

$\mathrm{T}=52 \mathrm{~ns}$

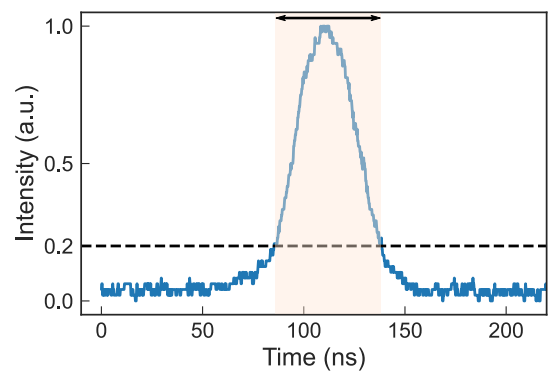

FIG. S15. Pulse shapes of the AOM. (a) Pulse duration $t_{D}=100$ ns: the rise time is 23 ns and the fall time is $25 \mathrm{~ns}$. (b) Pulse duration $t_{D}=50 \mathrm{~ns}$ : the full pulse duration measured is $52 \mathrm{~ns}$.

(a)

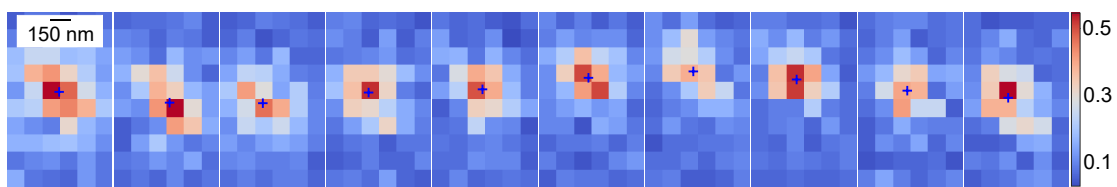

(b)

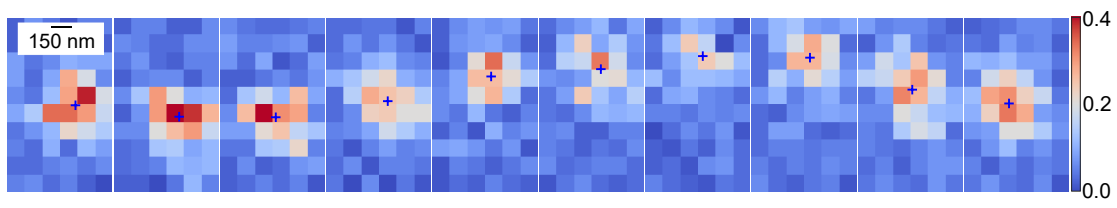

(c)

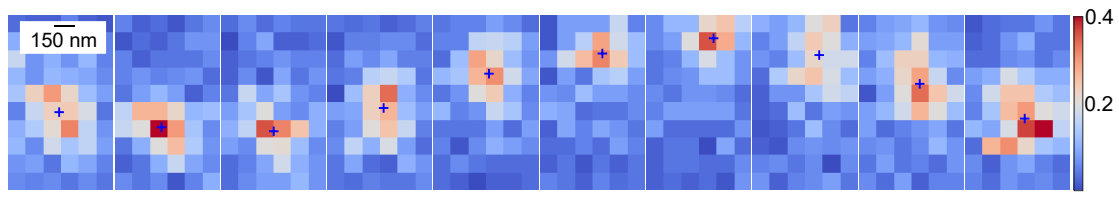

(d)

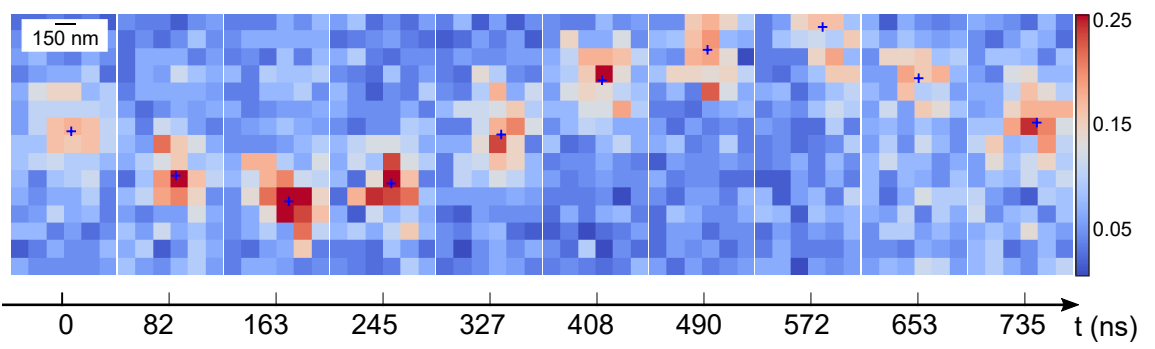

FIG. S16. Snapshots of ion's motion in a motion period under driving voltages $V_{\mathrm{pp}}=38 \mu \mathrm{V}$ (a), $82 \mu \mathrm{V}(\mathrm{b}), 122 \mu \mathrm{V}(\mathrm{c})$, and $216 \mu \mathrm{V}(\mathrm{d})$.

simply with the state rate equation

$$
\frac{d p_{0}}{d t}=-\beta \sigma \frac{I(x)}{\hbar \omega_{0}} p_{0}
$$

where $\beta=2 / 3$ is the branching ratio for the decay to bright states, $\sigma$ is the cross-section, $\omega_{0}$ is the angular frequency of the transition, and $I(x)$ is the intensity profile of the donut 


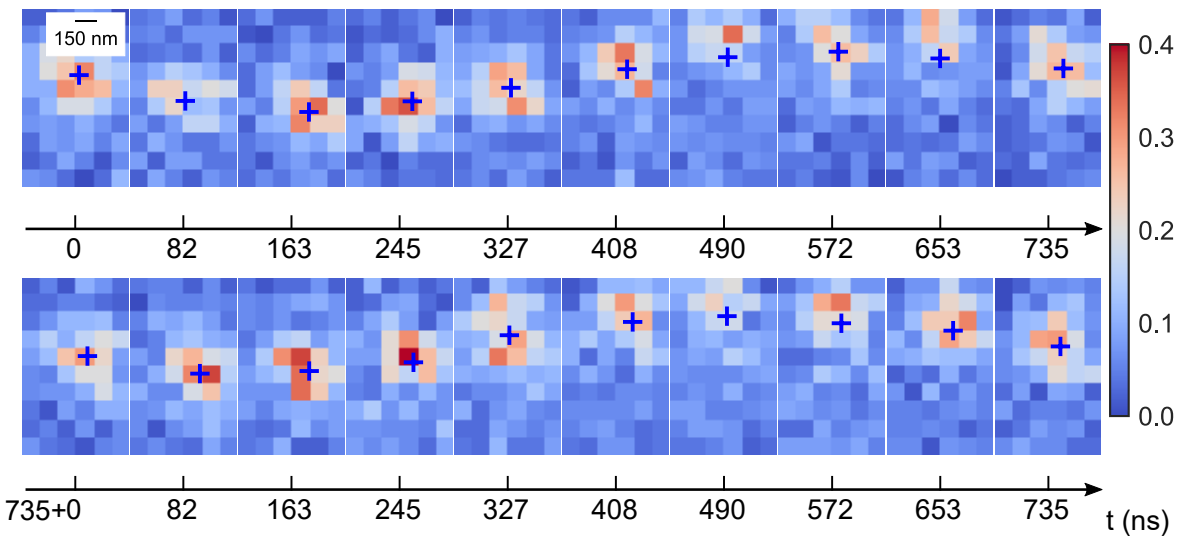

FIG. S17. Ion's trajectory of motion in two motion periods under a driving voltage $V_{\mathrm{pp}}=216 \mu \mathrm{V}$.

spot in terms of position $x$. For generality, we use the normalized intensity $s(x)=I(x) / I_{\text {sat }}$, where $I_{\text {sat }}=\hbar \omega_{0}^{3} \Gamma / 12 \pi c^{3}=510 \mathrm{~W} / \mathrm{m}^{2}$ is the saturation intensity. The solution of Eq. (S2) is given by

$$
p_{0}(x)=\exp \left[-\frac{\beta \sigma}{\hbar \omega_{0}} I_{\mathrm{sat}} s(x) t_{D}\right],
$$

where $k=\left(\beta \sigma / \hbar \omega_{0}\right) I_{\text {sat }}$. The depletion rate from dark state to bright state is $R_{x} \triangleq k s(x)$ and is proportional to the intensity. Therefore, Eq. (S3) can be written as

$$
p_{0}(x)=\exp \left[-k s(x) t_{D}\right]
$$

The normalized intensity profile of the donut beam ( $\mathrm{LG}_{0}^{1}$ beam) is

$$
s(x)=s_{0}+s_{\max }\left(x / r_{0}\right)^{2} \exp \left[1-\left(\frac{x}{r_{0}}\right)^{2}\right]
$$

where $r_{0}=\mathrm{FWHM}_{01}$, i.e., the full width at half maximum of the donut center, $s_{0}$ is the normalized residual intensity of the center, and $s_{\max }$ is the maximum intensity of the donut spot. The extinction ratio $\mathrm{ER}=s_{\max } / s_{0}$ is defined to characterize the ratio of the residual intensity of the center. In the low-intensity regime $\left(s_{\max } \ll 1\right)$, the bright-state probability $p_{1}=1-p_{0}$ is proportional to the depletion light intensity. Thus, the resolution $\Delta r$ is equal to $\mathrm{FWHM}_{01}$. In the high-intensity regime $\left(s_{\max } \gg 1\right)$, the nonlinear response of the ion to light makes the resolution $\Delta r \ll \mathrm{FWHM}_{01}$. Expansion of $s(x)$ at $x=0$ results in a parabolic shape:

$$
s(x) \approx s_{0}+e s_{\max }\left(\frac{x}{r_{0}}\right)^{2} .
$$


Therefore, the population of the dark state follows as

$$
\begin{aligned}
p_{0} & =\exp \left(-k s_{0} t_{D}\right) \exp \left[-k e s_{\max } t_{D}\left(\frac{x}{r_{0}}\right)^{2}\right] \\
& =\exp \left(-\frac{k s_{\max } t_{D}}{\mathrm{ER}}\right) \exp \left[-k e s_{\max } t_{D}\left(\frac{x}{r_{0}}\right)^{2}\right] .
\end{aligned}
$$

The first factor $p_{0}^{\max } \triangleq \exp \left(-k s_{\max } t_{D} / \mathrm{ER}\right)$ characterizes $p_{0}$ at the center of the donut spot, which will exponentially decline as $s_{0}$ increases. The resolution can be obtained from the second factor under the condition that this factor is equal to one-half, i.e., $\exp \left[-k e s_{\max } t_{D}\left(x_{0} / r_{0}\right)^{2}\right]=1 / 2$. Thus, the resolution is

$$
\Delta r=2 x_{0}=\sqrt{\frac{4 \ln 2}{e k s_{\max } t_{D}}} r_{0} \approx \frac{\mathrm{FWHM}_{01}}{\sqrt{k s_{\max } t_{D}}} .
$$

Uniting cases of low and high intensity, we obtain the formula for the resolution:

$$
\Delta r=\frac{\mathrm{FWHM}_{01}}{\sqrt{1+k s_{\max } t_{D}}},
$$

which is very similar to that in conventional STED microscopy [11].

The resolution is limited mainly by the normalized residual intensity $s_{0}$. When the signalto-noise ratio $\mathrm{SNR}=1$, i.e., $p_{0}^{\max }$ is equal to the error in dark-state measurement $\Delta p$, we can easily extract the resolution from $p_{0}$ :

$$
\exp \left(-\frac{k s_{\max } t_{D}}{\mathrm{ER}}\right)=\Delta p,
$$

and so

$$
k s_{\max } t_{D}=-\mathrm{ER} \ln \Delta p .
$$

Therefore, the resolution limit according to Eq. (S8) is

$$
\Delta r_{\text {limit }}=\frac{\mathrm{FWHM}_{01}}{\sqrt{-\mathrm{ER} \ln \Delta p}},
$$

and so $\Delta r_{\text {limit }} \propto \lambda / \mathrm{NA} \sqrt{\mathrm{ER}}$. For our experiment, the green dots in Fig. 2(f) of the main paper indicate that the measurement noise $\Delta p \approx 0.05, \mathrm{ER}=1 / 3.8 \%=26$, and $\Delta r_{\text {limit }}=159 \mathrm{~nm}$, which is in agreement with our measured best resolution of $175 \mathrm{~nm}$.

In regardless of the position drift of the experiment system, here we can give a theoretical resolution estimation of our method. In our current study, the aberration of the objective is large, from Fig. S7, we can find the phase difference between two adjacent pixels can almost 


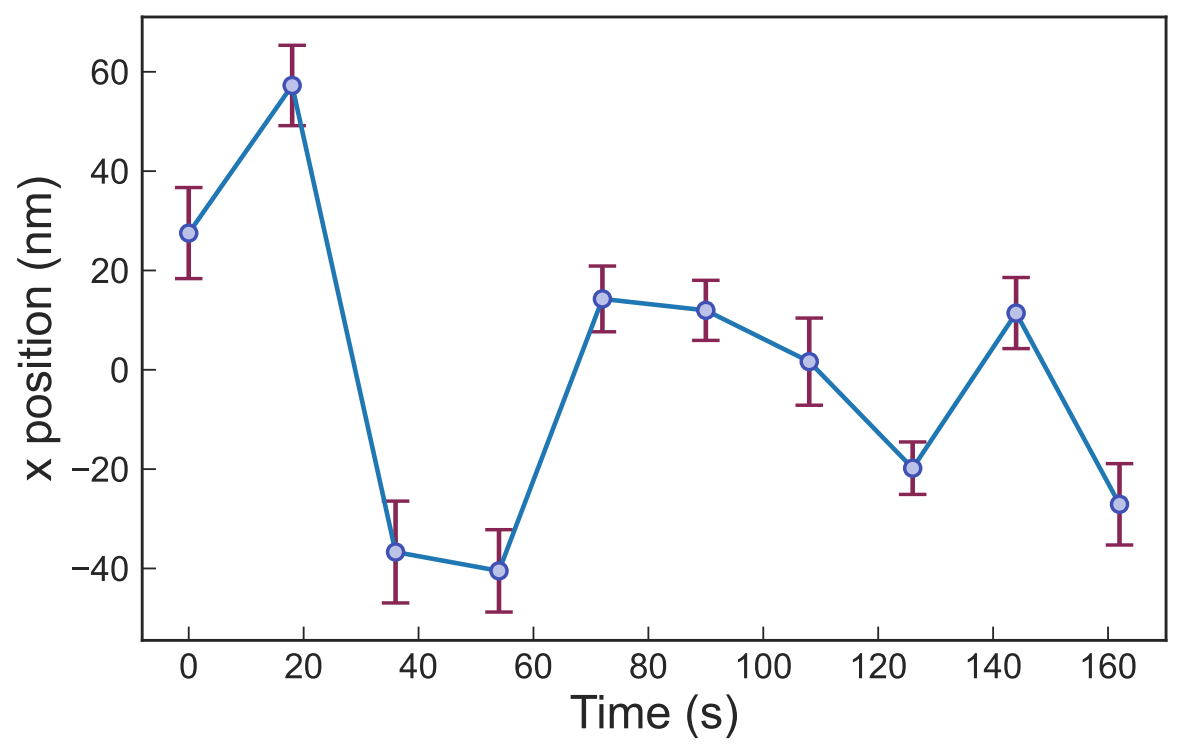

FIG. S18. Position drift of between the depletion spot and the ion. By tracking the ion in the $x$-direction (the axis is not driven) with different images in Fig. 3(c).

reach $\pi$ at the edge of the phase map, which means the real phase on the pixel is distributed in a range, not a fixed value, so the aberration on the pixel can not be compensated with a fix value. The large aberration of the objective makes the compensation failure at the edge of the pupil of the objective. If an objective with lower aberration and larger NA is used, e.g., $\mathrm{NA}=0.6$, the extinction ratio (ER) of the donut spot can be improved from 26 to 1000 $[4,12,13]$. From the deduced resolution relation in the supplementary Eq. S12, the resolution can be further improved to $4.7 \mathrm{~nm}$ with the NA and ER improvement. Furthermore, the fidelity of single-shot readout of the spin $\Delta p$ can be also improved with the high NA objective, from $5 \%$ to less than $0.1 \%$ [14], which will further improve the resolution to $3.1 \mathrm{~nm}$.

To realize the ultimate resolution, there will be lots of experimental challenges. A typical problem is the stability of the optical system. In our work, as the ion position displacement can be detected with $10 \mathrm{~nm}$ resolution, we can track the drift of the depletion light position by imaging the ion at different time. As taking each image in Fig. 3(c) needs 16 s, (the ion area just occupies several pixels in an image, so the imaging time on the ion area to get the displacement is about 1s, the time to obtain the ion displacement is so short that we can neglect the drift), we can get positions the ion in the $x$-direction (the axis is not driven) at different experiment time, which is shown in the Fig. S18, it reflects the position drift between the depletion spot and the ion. We can find the position drift is around $\pm 60 \mathrm{~nm}$ in 
$160 \mathrm{~s}$ and an average drift speed of $1.8 \mathrm{~nm} / \mathrm{s}$. It needed about $16 \mathrm{~s}$ to get the entire image in our current experiment, the drift during this time is about 29nm. We must note the estimated $29 \mathrm{~nm}$ is the drift after getting the entire image, as the ion image just occupied several pixels in the entire image, the drift during getting the ion image is much smaller in our experiment. By using a high NA objective to perform the experiment, the experiment time to get an image can be further compressed below $10 \mathrm{~s}$, because that the cooling time and detection time can be shortened from 1000us to 100us [14], so the drift between images can be further minimized.

Z.-H.Q. and J.-M.C. contributed equally to this work.

* jmcui@ustc.edu.cn

$\dagger$ hyf@ustc.edu.cn

$\ddagger$ cfli@ustc.edu.cn

[1] Z. Wang, L. Luo, K. Thadasina, K. Qian, J. Cui, and Y. Huang, EPJ Tech. Instrum. 3, 3 (2016).

[2] S. Olmschenk, K. C. Younge, D. L. Moehring, D. N. Matsukevich, P. Maunz, and C. Monroe, Phys. Rev. A 76, 052314 (2007).

[3] E. D. Black, Am. J. Phys. 69, 79 (2001).

[4] P. Zupancic, P. M. Preiss, R. Ma, A. Lukin, M. E. Tai, M. Rispoli, R. Islam, and M. Greiner, Opt. Express 24, 13881 (2016).

[5] C.-Y. Shih, S. Motlakunta, N. Kotibhaskar, M. Sajjan, R. Hablützel, and R. Islam, npj Quantum Inf. 7, 57 (2021).

[6] Y. Liu, Q. Zhang, and X. Su, Opt. Lasers Eng. 74, 22 (2015).

[7] J. Novák, P. Novák, and A. Mikš, Opt. Commun. 281, 5302 (2008).

[8] W.-H. Lee, Appl. Opt. 18, 3661 (1979).

[9] K. G. Johnson, J. D. Wong-Campos, A. Restelli, K. A. Landsman, B. Neyenhuis, J. Mizrahi, and C. Monroe, Rev. Sci. Instrum. 87, 053110 (2016).

[10] J. R. Johansson, P. D. Nation, and F. Nori, Comput. Phys. Commun. 184, 1234 (2013).

[11] V. Westphal and S. W. Hell, Phys. Rev. Lett. 94, 143903 (2005). 
[12] L. Yan, P. Gregg, E. Karimi, A. Rubano, L. Marrucci, R. Boyd, and S. Ramachandran, Optica 2, 900 (2015).

[13] L. Yan, P. Kristensen, and S. Ramachandran, APL Photonics 4, 022903 (2019).

[14] R. Noek, G. Vrijsen, D. Gaultney, E. Mount, T. Kim, P. Maunz, and J. Kim, Optics Letters 38, 4735 (2013). 